\title{
Amplitude analysis and branching fraction measurement of $\bar{B}_{s}^{0} \rightarrow J / \psi K^{+} K^{-}$
}

\author{
R. Aaij et al.* \\ (LHCb Collaboration) \\ (Received 5 February 2013; published 8 April 2013)
}

\begin{abstract}
An amplitude analysis of the final state structure in the $\bar{B}_{s}^{0} \rightarrow J / \psi K^{+} K^{-}$decay mode is performed using $1.0 \mathrm{fb}^{-1}$ of data collected by the LHCb experiment in $7 \mathrm{TeV}$ center-of-mass energy $p p$ collisions produced by the LHC. A modified Dalitz plot analysis of the final state is performed using both the invariant mass spectra and the decay angular distributions. Resonant structures are observed in the $K^{+} K^{-}$mass spectrum as well as a significant nonresonant $S$-wave contribution over the entire $K^{+} K^{-}$mass range. The largest resonant component is the $\phi(1020)$, accompanied by $f_{0}(980), f_{2}^{\prime}(1525)$, and four additional resonances. The overall branching fraction is measured to be $\mathcal{B}\left(\bar{B}_{s}^{0} \rightarrow J / \psi K^{+} K^{-}\right)=(7.70 \pm 0.08 \pm 0.39 \pm 0.60) \times 10^{-4}$, where the first uncertainty is statistical, the second systematic, and the third due to the ratio of the number of $\bar{B}_{s}^{0}$ to $B^{-}$mesons produced. The mass and width of the $f_{2}^{\prime}(1525)$ are measured to be $1522.2 \pm 2.8_{-2.0}^{+5.3} \mathrm{MeV}$ and $84 \pm 6_{-5}^{+10} \mathrm{MeV}$, respectively. The final state fractions of the other resonant states are also reported.
\end{abstract}

DOI: 10.1103/PhysRevD.87.072004

PACS numbers: $14.40 . \mathrm{Nd}, 13.20 . \mathrm{He}, 14.40 . \mathrm{Be}$

\section{INTRODUCTION}

The study of $\bar{B}_{s}^{0}$ decays to $J / \psi h^{+} h^{-}$, where $h$ is either a pion or kaon, has been used to measure mixing-induced $C P$ violation in $\bar{B}_{s}^{0}$ decays $[1-7] .{ }^{1}$ In order to best exploit these decays, a better understanding of the final state composition is necessary. This study has been reported for the $\bar{B}_{s}^{0} \rightarrow$ $J / \psi \pi^{+} \pi^{-}$channel [8]. Here we perform a similar analysis for $\bar{B}_{s}^{0} \rightarrow J / \psi K^{+} K^{-}$. While a large $\phi(1020)$ contribution is well-known [9] and the $f_{2}^{\prime}(1525)$ component has been recently observed [10] and confirmed [11], other components have not heretofore been identified including the sources of $S$-wave contributions [12]. The tree-level Feynman diagram for the process is shown in Fig. 1.

In this paper the $J / \psi K^{+}$and $K^{+} K^{-}$mass spectra and decay angular distributions are used to study resonant and nonresonant structures. This differs from a classical "Dalitz plot" analysis [13] since the $J / \psi$ meson has spin- 1 , and its three helicity amplitudes must be considered.

\section{DATA SAMPLE AND DETECTOR}

The event sample is obtained using $1.0 \mathrm{fb}^{-1}$ of integrated luminosity collected with the LHCb detector [14] using $p p$ collisions at a center-of-mass energy of $7 \mathrm{TeV}$. The detector is a single-arm forward spectrometer covering the pseudorapidity range $2<\eta<5$, designed for the study of particles containing $b$ or $c$ quarks. Components include a high-precision tracking system consisting of a silicon-strip

*Full author list given at the end of the article.

Published by the American Physical Society under the terms of the Creative Commons Attribution 3.0 License. Further distribution of this work must maintain attribution to the author(s) and the published article's title, journal citation, and DOI.

${ }^{1}$ Mention of a particular mode implies use of its charge conjugate throughout this paper. vertex detector surrounding the $p p$ interaction region, a large-area silicon strip detector located upstream of a dipole magnet with a bending power of about $4 \mathrm{Tm}$, and three stations of silicon strip detectors and straw drift tubes placed downstream. The combined tracking system has momentum $^{2}$ resolution $\Delta p / p$ that varies from $0.4 \%$ at $5 \mathrm{GeV}$ to $0.6 \%$ at $100 \mathrm{GeV}$. The impact parameter (IP) is defined as the minimum distance of approach of the track with respect to the primary vertex. For tracks with large transverse momentum with respect to the proton beam direction, the IP resolution is approximately $20 \mu \mathrm{m}$. Charged hadrons are identified using two ring-imaging Cherenkov detectors. Photon, electron, and hadron candidates are identified by a calorimeter system consisting of scintillating-pad and preshower detectors, an electromagnetic calorimeter, and a hadronic calorimeter. Muons are identified by a system composed of alternating layers of iron and multiwire proportional chambers.

The trigger [15] consists of a hardware stage, based on information from the calorimeter and muon systems, followed by a software stage that applies a full event reconstruction. Events selected for this analysis are triggered by a $J / \psi \rightarrow$ $\mu^{+} \mu^{-}$decay, where the $J / \psi$ is required at the software level to be consistent with coming from the decay of a $\bar{B}_{s}^{0}$ meson by use either of IP requirements or detachment of the $J / \psi$ from the primary vertex. Monte Carlo simulations are performed using PYTHIA [16] with the specific tuning given in Ref. [17], and the LHCb detector description based on GEANT4 [18] described in Ref. [19]. Decays of $B$ mesons are based on EVTGEN [20].

\section{SIGNAL SELECTION AND BACKGROUNDS}

We select $\bar{B}_{s}^{0} \rightarrow J / \psi K^{+} K^{-}$candidates trying to simultaneously maximize the signal yield and reduce the

\footnotetext{
${ }^{2}$ We work in units where $c=1$.
} 


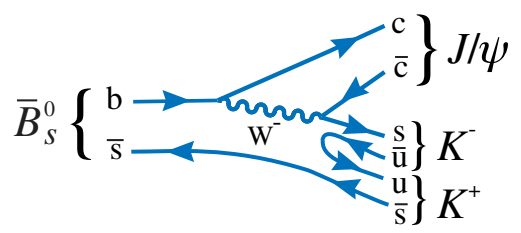

FIG. 1 (color online). Leading order diagram for $\bar{B}_{s}^{0} \rightarrow$ $J / \psi K^{+} K^{-}$.

background. Candidate $J / \psi \rightarrow \mu^{+} \mu^{-}$decays are combined with a pair of kaon candidates of opposite charge and then required that all four tracks are consistent with coming from a common decay point. To be considered a $J / \psi \rightarrow \mu^{+} \mu^{-}$candidate, particles identified as muons of opposite charge are required to have transverse momentum, $p_{\mathrm{T}}$, greater than $500 \mathrm{MeV}$ and form a vertex with fit $\chi^{2}$ per number of degrees of freedom (ndf) less than 11. These requirements give rise to a large $J / \psi$ signal over a small background [21]. Only candidates with a dimuon invariant mass between $-48 \mathrm{MeV}$ to $+43 \mathrm{MeV}$ relative to the observed $J / \psi$ mass peak are selected. The asymmetric requirement is due to final-state electromagnetic radiation. The two muons are subsequently kinematically constrained to the known $J / \psi$ mass [9].

Our ring-imaging Cherenkov system allows for the possibility of positively identifying kaon candidates. Charged tracks produce Cherenkov photons whose emission angles are compared with those expected for electrons, pions, kaons, or protons and a likelihood for each species is then computed. To identify a particular species, the difference between the logarithm of the likelihoods for two particle hypotheses (DLL) is computed. There are two criteria used: loose corresponds to $\operatorname{DLL}(K-\pi)>0$, while tight has $\operatorname{DLL}(K-\pi)>10$ and $\operatorname{DLL}(K-p)>-3$. Unless stated otherwise, we require the tight criterion for kaon selection.

We select candidate $K^{+} K^{-}$combinations if each particle is inconsistent with having been produced at the primary vertex. For this test we require that the $\chi^{2}$ formed by using the hypothesis that the IP is zero be greater than 9 for each track. Furthermore, each kaon must have $p_{\mathrm{T}}>$ $250 \mathrm{MeV}$ and the scalar sum of the $p_{\mathrm{T}}$ of the kaon candidates must be greater than $900 \mathrm{MeV}$. To select $\bar{B}_{s}^{0}$ candidates, we further require that the two kaon candidates form a vertex with $\chi^{2}<10$, and that they form a candidate $\bar{B}_{s}^{0}$ vertex with the $J / \psi$ where the vertex fit $\chi^{2} /$ ndf $<5$. We require that this $\bar{B}_{s}^{0}$ vertex be more than $1.5 \mathrm{~mm}$ from the primary vertex, and the angle between the $\bar{B}_{s}^{0}$ momentum vector and the vector from the primary vertex to the $\bar{B}_{s}^{0}$ vertex must be less than $11.8 \mathrm{mrad}$.

The $\bar{B}_{s}^{0}$ candidate invariant mass distribution is shown in Fig. 2. The vertical lines indicate the signal and sideband regions, where the signal region extends to $\pm 20 \mathrm{MeV}$ around the nominal $\bar{B}_{s}^{0}$ mass [9] and the sidebands extend from $35 \mathrm{MeV}$ to $60 \mathrm{MeV}$ on either side of the peak. The small peak near $5280 \mathrm{MeV}$ results from $\bar{B}^{0}$ decays and will be subject to future investigation.

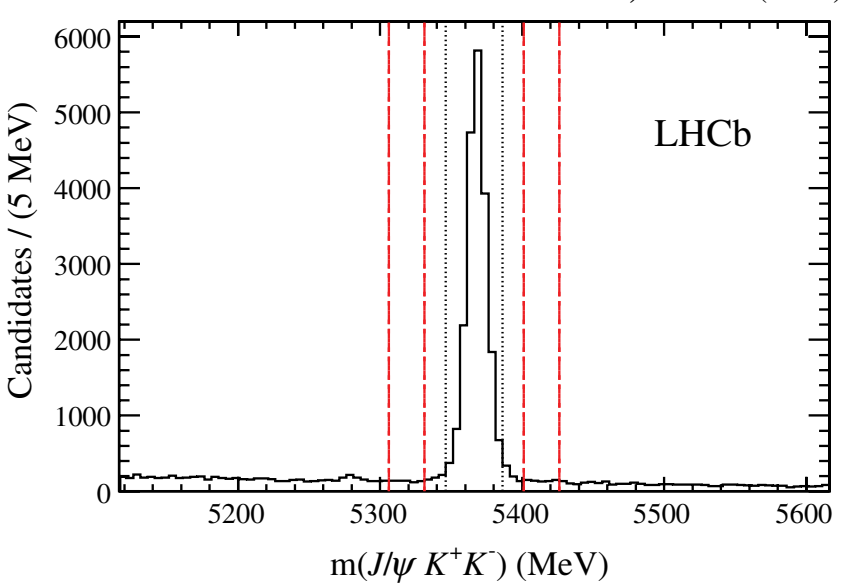

FIG. 2 (color online). Invariant mass spectrum of $J / \psi K^{+} K^{-}$ combinations. The vertical lines indicate the signal dotted (black) and sideband dashed (red) regions.

The background consists of combinations of tracks, which have a smooth mass shape through the $J / \psi K^{+} K^{-}$ region and peaking contributions caused by the reflection of specific decay modes where a pion is misidentified as a kaon. The reflection background that arises from the decay $\bar{B}^{0} \rightarrow J / \psi K^{-} \pi^{+}$, where the $\pi^{+}$is misidentified as a $K^{+}$, is determined from the number of $\bar{B}^{0}$ candidates in the control region 25-200 MeV above the $\bar{B}_{s}^{0}$ mass peak.

For each of the candidates in the $J / \psi K^{+} K^{-}$control region, we reassign each of the two kaons in turn to the pion mass hypothesis. The resulting $J / \psi K \pi$ invariant mass distribution is shown in Fig. 3. The peak at the $\bar{B}^{0}$ mass has $906 \pm 51$ candidates, determined by fitting the data to a Gaussian function for the signal, and a polynomial function for the background. From these events we estimate the number in the $\bar{B}_{s}^{0}$ signal region, based on a simulation of the shape of the reflected distribution as a

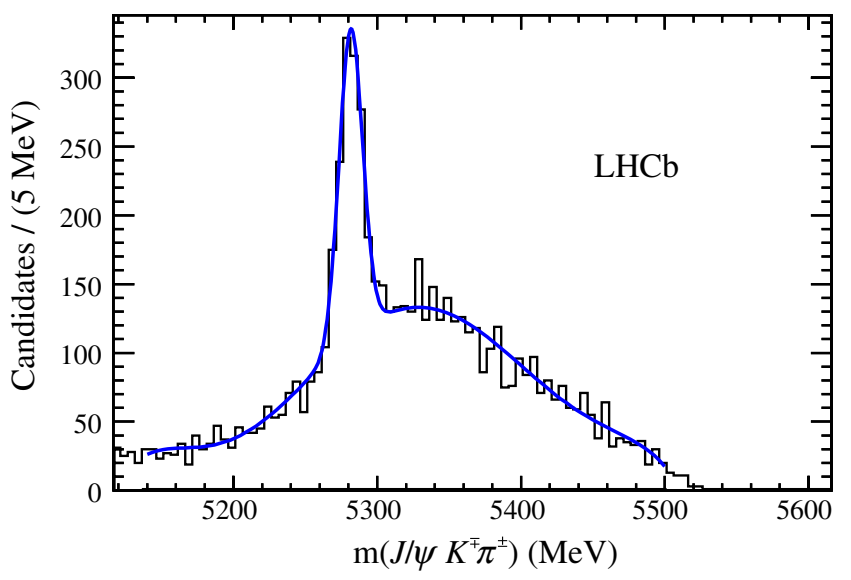

FIG. 3 (color online). Invariant mass distribution for $J / \psi K^{+} K^{-}$candidates $25-200 \mathrm{MeV}$ above the $\bar{B}_{s}^{0}$ mass, reinterpreted as $\bar{B}^{0} \rightarrow J / \psi K^{\overline{+}} \pi^{ \pm}$events. The fit is to a signal Gaussian whose mass and width are allowed to vary as well as a polynomial background. 


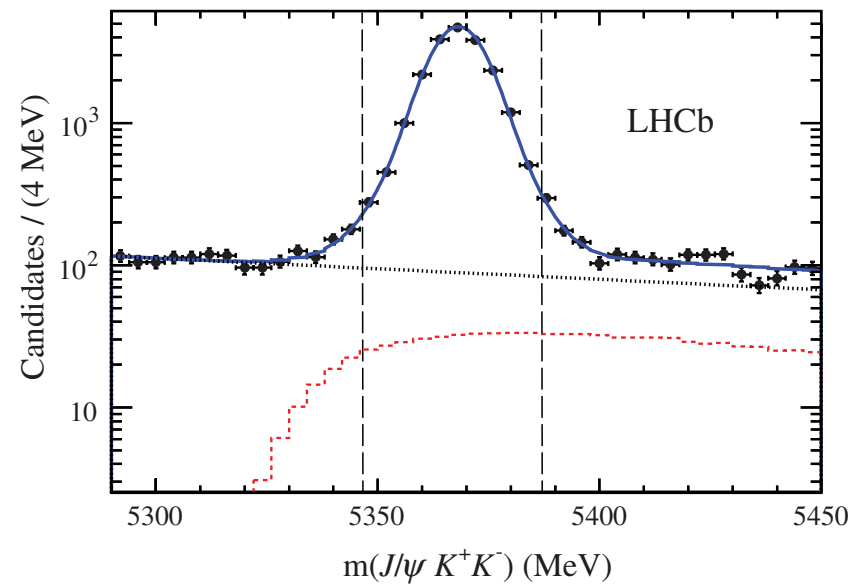

FIG. 4 (color online). Fit to the invariant mass spectrum of $J / \psi K^{+} K^{-}$combinations. The dotted line (black) is the combinatorial background, the dashed shape (red) shows the misidentified $\bar{B}^{0} \rightarrow J / \psi K^{-} \pi^{+}$decays, and the solid curve (blue) shows the total. The vertical dashed lines indicate the signal region.

function of $J / \psi K^{-} K^{+}$mass. Using simulated $\bar{B}^{0} \rightarrow$ $J / \psi \bar{K}^{* 0}(892)$ and $\bar{B}^{0} \rightarrow J / \psi \bar{K}_{2}^{*}(1430)$ samples, we calculate $309 \pm 17$ reflection candidates within $\pm 20 \mathrm{MeV}$ of the $\bar{B}_{s}^{0}$ peak. This number is used as a constraint in the mass fit described below.

To determine the number of $\bar{B}_{s}^{0}$ signal candidates we perform a fit to the candidate $J / \psi K^{+} K^{-}$invariant mass spectrum shown in Fig. 4. The fit function is the sum of the $\bar{B}_{s}^{0}$ signal component, combinatorial background, and the contribution from the $\bar{B}^{0} \rightarrow J / \psi K^{-} \pi^{+}$reflections. The signal is modeled by a double-Gaussian function with a common mean. The combinatorial background is described by a linear function. The reflection background is constrained as described above. The mass fit gives 19, $195 \pm 150$ signal together with $894 \pm 24$ combinatorial background candidates within $\pm 20 \mathrm{MeV}$ of the $\bar{B}_{s}^{0}$ mass peak.

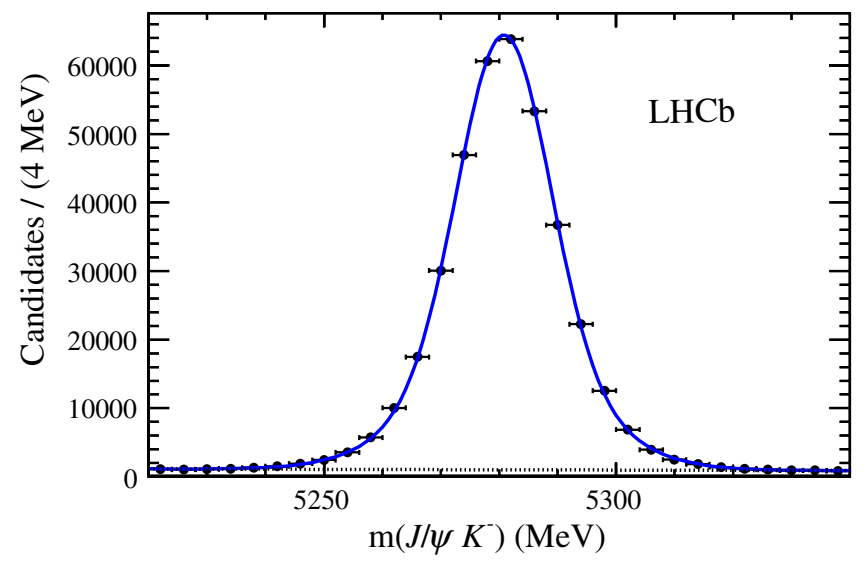

FIG. 5 (color online). Fit to the invariant mass spectrum of $J / \psi K^{-}$candidates. The dotted line shows the combinatorial background and the solid curve (blue) is the total.
We use the decay $B^{-} \rightarrow J / \psi K^{-}$as the normalization channel for branching fraction determinations. The selection criteria are similar to those used for $J / \psi K^{+} K^{-}$, except for particle identification as here a loose kaon identification criterion is used. Figure 5 shows the $J / \psi K^{-}$mass distribution. The signal is fit with a double-Gaussian function and a linear function is used to fit the combinatorial background. There are 342, $786 \pm$ 661 signal and 10,195 \pm 134 background candidates within $\pm 20 \mathrm{MeV}$ of the $B^{-}$peak.

\section{ANALYSIS FORMALISM}

One of the goals of this analysis is to determine the intermediate states in $\bar{B}_{s}^{0} \rightarrow J / \psi K^{+} K^{-}$decay within the context of an isobar model [22,23], where we sum the resonant and nonresonant components testing if they explain the invariant mass squared and angular distributions. We also determine the absolute branching fractions of $\bar{B}_{s}^{0} \rightarrow J / \psi \phi(1020)$ and $\bar{B}_{s}^{0} \rightarrow J / \psi f_{2}^{\prime}(1525)$ final states and the mass and width of the $f_{2}^{\prime}(1525)$ resonance. Another important goal is to understand the $S$-wave content in the $\phi(1020)$ mass region.

Four variables completely describe the decay of $\bar{B}_{s}^{0} \rightarrow$ $J / \psi K^{+} K^{-}$with $J / \psi \rightarrow \mu^{+} \mu^{-}$. Two are the invariant mass squared of $J / \psi K^{+}, s_{12} \equiv m^{2}\left(J / \psi K^{+}\right)$, and the invariant mass squared of $K^{+} K^{-}, s_{23} \equiv m^{2}\left(K^{+} K^{-}\right)$. The other two are the $J / \psi$ helicity angle, $\theta_{J / \psi}$, which is the angle of the $\mu^{+}$in the $J / \psi$ rest frame with respect to the $J / \psi$ direction in the $\bar{B}_{s}^{0}$ rest frame, and the angle between the $J / \psi$ and $K^{+} K^{-}$decay planes, $\chi$, in the $\bar{B}_{s}^{0}$ rest frame. To simplify the probability density function (PDF), we analyze the decay process after integrating over the angular variable $\chi$, which eliminates several interference terms.

\section{A. The model for $\bar{B}_{s}^{\mathbf{0}} \rightarrow J / \boldsymbol{\psi} K^{+} K^{-}$}

In order to perform an amplitude analysis a PDF must be constructed that models correctly the dynamical and kinematic properties of the decay. The PDF is separated into two components, one describing signal, $S$, and the other background, $B$. The overall PDF given by the sum is

$$
\begin{aligned}
F\left(s_{12}, s_{23}, \theta_{J / \psi}\right)= & \frac{1-f_{\text {com }}-f_{\text {refl }}}{\mathcal{N}_{\text {sig }}} \varepsilon\left(s_{12}, s_{23}, \theta_{J / \psi}\right) \\
& \times S\left(s_{12}, s_{23}, \theta_{J / \psi}\right)+B\left(s_{12}, s_{23}, \theta_{J / \psi}\right),
\end{aligned}
$$

where $\varepsilon$ is the detection efficiency. The background is described by the sum of combinatorial background, $C$, and reflection, $R$, functions

$$
\begin{aligned}
B\left(s_{12}, s_{23}, \theta_{J / \psi}\right)= & \frac{f_{\text {com }}}{\mathcal{N}_{\text {com }}} C\left(s_{12}, s_{23}, \theta_{J / \psi}\right) \\
& +\frac{f_{\text {refl }}}{\mathcal{N}_{\text {refl }}} R\left(s_{12}, s_{23}, \theta_{J / \psi}\right),
\end{aligned}
$$


where $f_{\text {com }}$ and $f_{\text {refl }}$ are the fractions of the combinatorial background and reflection, respectively, in the fitted region. The fractions $f_{\text {com }}$ and $f_{\text {refl }}$ obtained from the mass fit are fixed for the subsequent analysis.

The normalization factors are given by

$$
\begin{aligned}
\mathcal{N}_{\text {sig }}= & \int \varepsilon\left(s_{12}, s_{23}, \theta_{J / \psi}\right) S\left(s_{12}, s_{23}, \theta_{J / \psi}\right) \\
& \times d s_{12} d s_{23} d \cos \theta_{J / \psi}, \\
\mathcal{N}_{\text {com }}= & \int C\left(s_{12}, s_{23}, \theta_{J / \psi}\right) d s_{12} d s_{23} d \cos \theta_{J / \psi}, \\
\mathcal{N}_{\text {refl }}= & \int R\left(s_{12}, s_{23}, \theta_{J / \psi}\right) d s_{12} d s_{23} d \cos \theta_{J / \psi}
\end{aligned}
$$

This formalism is similar to that used by Belle in their analysis of $\bar{B}^{0} \rightarrow K^{-} \pi^{+} \chi_{c 1}$ [24], and later used by LHCb for the analysis of $\bar{B}_{s}^{0} \rightarrow J / \psi \pi^{+} \pi^{-}$[8].

The invariant mass squared of $J / \psi K^{+}$versus $K^{+} K^{-}$ is shown in Fig. 6 for $\bar{B}_{s}^{0} \rightarrow J / \psi K^{+} K^{-}$candidates. No structure is seen in $m^{2}\left(J / \psi K^{+}\right)$. There are however visible horizontal bands in the $K^{+} K^{-}$mass squared spectrum, the most prominent of which correspond to the $\phi(1020)$ and $f_{2}^{\prime}(1525)$ resonances. These and other structures in $m^{2}\left(K^{+} K^{-}\right)$are now examined.

The signal function is given by the coherent sum over resonant states that decay into $K^{+} K^{-}$, plus a possible nonresonant $S$-wave contribution ${ }^{3}$

$S\left(s_{12}, s_{23}, \theta_{J / \psi}\right)=\sum_{\lambda=0, \pm 1}\left|\sum_{i} a_{\lambda}^{R_{i}} e^{i \phi_{\lambda}^{R_{i}}} \mathcal{A}_{\lambda}^{R_{i}}\left(s_{12}, s_{23}, \theta_{J / \psi}\right)\right|^{2}$,

where $\mathcal{A}_{\lambda}^{R_{i}}\left(s_{12}, s_{23}, \theta_{J / \psi}\right)$ describes the decay amplitude via an intermediate resonance state $R_{i}$ with helicity $\lambda$. Note that the $J / \psi$ has the same helicity as the intermediate $K^{+} K^{-}$resonance. Each $R_{i}$ has an associated amplitude strength $a_{\lambda}^{R_{i}}$ and a phase $\phi_{\lambda}^{R_{i}}$ for each helicity state $\lambda$. The amplitude for resonance $R$, for each $i$, is given by

$$
\begin{aligned}
\mathcal{A}_{\lambda}^{R}\left(s_{12}, s_{23}, \theta_{J / \psi}\right)= & F_{B}^{\left(L_{B}\right)} A_{R}\left(s_{23}\right) F_{R}^{\left(L_{R}\right)} T_{\lambda}\left(\theta_{K K}\right)\left(\frac{P_{B}}{m_{B}}\right)^{L_{B}} \\
& \times\left(\frac{P_{R}}{\sqrt{s_{23}}}\right)^{L_{R}} \Theta_{\lambda}\left(\theta_{J / \psi}\right),
\end{aligned}
$$

where $P_{R}$ is the momentum of either of the two kaons in the di-kaon rest frame, $m_{B}$ is the $\bar{B}_{s}^{0}$ mass, $P_{B}$ is the magnitude of the $J / \psi$ three-momentum in the $\bar{B}_{s}^{0}$ rest frame, and $F_{B}^{\left(L_{B}\right)}$ and $F_{R}^{\left(L_{R}\right)}$ are the $\bar{B}_{s}^{0}$ meson and $R_{i}$ resonance decay form factors. The orbital angular momenta between the $J / \psi$ and $K^{+} K^{-}$system is given by $L_{B}$, and the orbital angular momentum in the $K^{+} K^{-}$decay is given by $L_{R}$; the latter

\footnotetext{
${ }^{3}$ The interference terms between different helicities are zero because we integrate over the angular variable $\chi$.
}

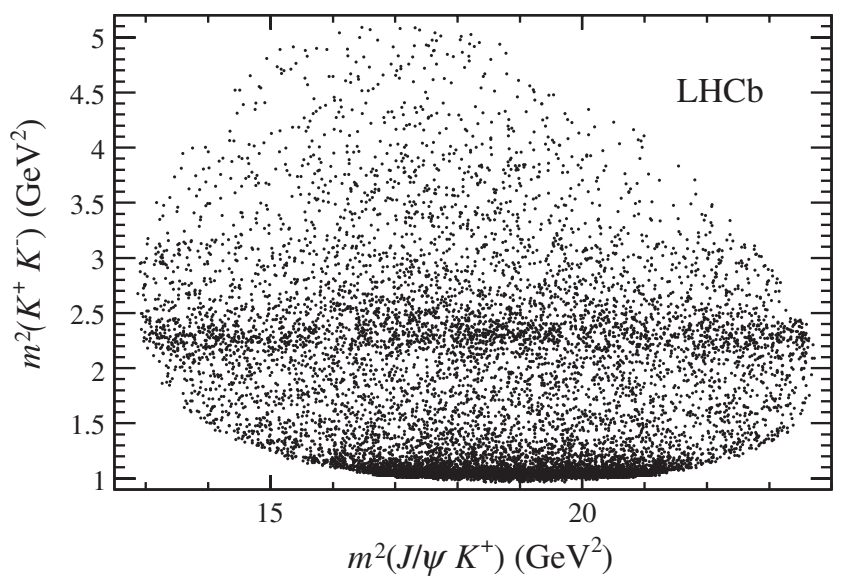

FIG. 6. Distribution of $m^{2}\left(K^{+} K^{-}\right)$versus $m^{2}\left(J / \psi K^{+}\right)$ for $\bar{B}_{s}^{0}$ candidate decays within $\pm 20 \mathrm{MeV}$ of the $\bar{B}_{s}^{0}$ mass. The horizontal bands result from the $\phi(1020)$ and $f_{2}^{\prime}(1525)$ resonances.

is the same as the spin of the $K^{+} K^{-}$system. Since the parent $\bar{B}_{s}^{0}$ has spin- 0 and the $J / \psi$ is a vector, when the $K^{+} K^{-}$system forms a spin-0 resonance, $L_{B}=1$ and $L_{R}=0$. For $K^{+} K^{-}$resonances with nonzero spin, $L_{B}$ can be 0,1 or $2(1,2$ or 3$)$ for $L_{R}=1(2)$ and so on. We take the lowest $L_{B}$ as the default value and consider the other possibilities in the systematic uncertainty.

The Blatt-Weisskopf barrier factors $F_{B}^{\left(L_{B}\right)}$ and $F_{R}^{\left(L_{R}\right)}$ [25] are

$$
\begin{aligned}
& F^{(0)}=1, \quad F^{(1)}=\frac{\sqrt{1+z_{0}}}{\sqrt{1+z}}, \\
& F^{(2)}=\frac{\sqrt{z_{0}^{2}+3 z_{0}+9}}{\sqrt{z^{2}+3 z+9}} .
\end{aligned}
$$

For the $B$ meson $z=r^{2} P_{B}^{2}$, where $r$, the hadron scale, is taken as $5.0 \mathrm{GeV}^{-1}$; for the $R$ resonance $z=r^{2} P_{R}^{2}$, and $r$ is taken as $1.5 \mathrm{GeV}^{-1}$ [26]. In both cases $z_{0}=r^{2} P_{0}^{2}$ where $P_{0}$ is the decay daughter momentum at the pole mass; for the $\bar{B}_{s}^{0}$ decay the $J / \psi$ momentum is used, while for the $R$ resonances the kaon momentum is used.

In the helicity formalism, the angular term, $T_{\lambda}\left(\theta_{K K}\right)$ is defined as

$$
T_{\lambda}\left(\theta_{K K}\right)=d_{\lambda 0}^{J}\left(\theta_{K K}\right),
$$

where $d$ is the Wigner $d$-function, $J$ is the resonance spin, $\theta_{K K}$ is the helicity angle of the $K^{+}$in the $K^{+} K^{-}$rest frame with respect to the $K^{+} K^{-}$direction in the $\bar{B}_{s}^{0}$ rest frame and may be calculated directly from the other variables as

$\cos \theta_{K K}=\frac{\left[m^{2}\left(J / \psi K^{+}\right)-m^{2}\left(J / \psi K^{-}\right)\right] m\left(K^{+} K^{-}\right)}{4 P_{R} P_{B} m_{B}}$.

The $J / \psi$ helicity dependent term $\Theta_{\lambda}\left(\theta_{J / \psi}\right)$ is defined as 


$$
\begin{aligned}
\Theta_{\lambda}\left(\theta_{J / \psi}\right) & =\sqrt{\sin ^{2} \theta_{J / \psi}} & \text { for } \lambda=0 \\
& =\sqrt{\frac{1+\cos ^{2} \theta_{J / \psi}}{2}} & \text { for }|\lambda|=1 .
\end{aligned}
$$

The mass squared shape of each resonance, $R$ is described by the function $A_{R}\left(s_{23}\right)$. In most cases this is a Breit-Wigner (BW) amplitude. When a decay channel opens close to the resonant mass, complications arise since the proximity of the second threshold distorts the line shape of the amplitude. The $f_{0}(980)$ can decay to either $\pi \pi$ or $K K$. While the $\pi \pi$ channel opens at much lower masses, the $K^{+} K^{-}$decay channel opens near the resonance mass. Thus, for the $f_{0}(980)$ we use a Flatté model [27] that takes into account these coupled channels.

We describe the BW amplitude for a resonance decaying into two spin- 0 particles, labeled as 2 and 3 , as

$$
A_{R}\left(s_{23}\right)=\frac{1}{m_{R}^{2}-s_{23}-i m_{R} \Gamma\left(s_{23}\right)},
$$

where $m_{R}$ is the resonance mass, $\Gamma\left(s_{23}\right)$ is its energydependent width that is parametrized as

$$
\Gamma\left(s_{23}\right)=\Gamma_{0}\left(\frac{P_{R}}{P_{R_{0}}}\right)^{2 L_{R}+1}\left(\frac{m_{R}}{\sqrt{s_{23}}}\right) F_{R}^{2} .
$$

Here $\Gamma_{0}$ is the decay width when the invariant mass of the daughter combinations is equal to $m_{R}$.

The Flatté mass shape is parametrized as

$$
A_{R}\left(s_{23}\right)=\frac{1}{m_{R}^{2}-s_{23}-i m_{R}\left(g_{\pi \pi} \rho_{\pi \pi}+g_{K K} \rho_{K K}\right)},
$$

where the constants $g_{\pi \pi}$ and $g_{K K}$ are the $f_{0}(980)$ couplings to $\pi^{+} \pi^{-}$and $K^{+} K^{-}$final states, respectively. The $\rho$ factors are given by Lorentz-invariant phase space

$$
\begin{gathered}
\rho_{\pi \pi}=\frac{2}{3} \sqrt{1-\frac{4 m_{\pi^{ \pm}}^{2}}{s_{23}}}+\frac{1}{3} \sqrt{1-\frac{4 m_{\pi^{0}}^{2}}{s_{23}}}, \\
\rho_{K K}=\frac{1}{2} \sqrt{1-\frac{4 m_{K^{ \pm}}^{2}}{s_{23}}}+\frac{1}{2} \sqrt{1-\frac{4 m_{K^{0}}^{2}}{s_{23}}} .
\end{gathered}
$$

For nonresonant processes, the amplitude $\mathcal{A}\left(s_{12}, s_{23}, \theta_{J / \psi}\right)$ is constant over the variables $s_{12}$ and $s_{23}$ but has an angular dependence due to the $J / \psi$ decay. The amplitude is derived from Eq. (5), assuming that the nonresonant $K^{+} K^{-}$contribution is a $S$-wave (i.e., $L_{R}=0$, $L_{B}=1$ ) and is uniform in phase space (i.e., $A_{R}=1$ ),

$$
\mathcal{A}\left(s_{12}, s_{23}, \theta_{J / \psi}\right)=\frac{P_{B}}{m_{B}} \sqrt{\sin ^{2} \theta_{J / \psi}} \text {. }
$$

\section{B. Detection efficiency}

The detection efficiency is determined from a phase space simulation sample containing $3.4 \times 10^{6}$ $\bar{B}_{s}^{0} \rightarrow J / \psi K^{+} K^{-}$events with $J / \psi \rightarrow \mu^{+} \mu^{-}$. We also use a separate sample of $1.3 \times 10^{6} \bar{B}_{s}^{0} \rightarrow J / \psi \phi$ events. The $p$ and $p_{\mathrm{T}}$ distributions of the generated $\bar{B}_{s}^{0}$ mesons are weighted to match the distributions found using $J / \psi \phi$ data. The simulation is also corrected by weighting for difference between the simulated kaon detection efficiencies and the measured ones determined by using a sample of $D^{*+} \rightarrow \pi^{+}\left(D^{0} \rightarrow K^{-} \pi^{+}\right)$events.

Next we describe the efficiency in terms of the analysis variables. Both $s_{12}$ and $s_{13}$ range from $12.5 \mathrm{GeV}^{2}$ to $24.0 \mathrm{GeV}^{2}$, where $s_{13}$ is defined below, and thus are centered at $s_{0}=18.25 \mathrm{GeV}^{2}$. We model the detection efficiency using the dimensionless symmetric Dalitz plot observables

$x=\left(s_{12}-s_{0}\right) /\left(1 \mathrm{GeV}^{2}\right), \quad y=\left(s_{13}-s_{0}\right) /\left(1 \mathrm{GeV}^{2}\right)$,

and the angular variable $\theta_{J / \psi}$. The observables $s_{12}$ and $s_{13}$ are related to $s_{23}$ as

$$
s_{12}+s_{13}+s_{23}=m_{B}^{2}+m_{J / \psi}^{2}+m_{K^{+}}^{2}+m_{K^{-}}^{2} .
$$

To parametrize this efficiency, we fit the $\cos \theta_{J / \psi}$ distributions of the $J / \psi K^{+} K^{-}$and $J / \psi \phi$ simulation samples in bins of $m^{2}\left(K^{+} K^{-}\right)$with the function

$$
\varepsilon_{2}\left(s_{23}, \theta_{J / \psi}\right)=\frac{1+a \cos ^{2} \theta_{J / \psi}}{2+2 a / 3},
$$

giving values of $a$ as a function of $m^{2}\left(K^{+} K^{-}\right)$. The resulting distribution, shown in Fig. 7, is described by an exponential function

$$
a\left(s_{23}\right)=\exp \left(a_{1}+a_{2} s_{23}\right),
$$

with $a_{1}=-0.76 \pm 0.18$ and $a_{2}=(-1.02 \pm 0.15) \mathrm{GeV}^{-2}$. Equation (18) is normalized with respect to $\cos \theta_{J / \psi}$. The efficiency in $\cos \theta_{J / \psi}$ depends on $s_{23}$, and is observed

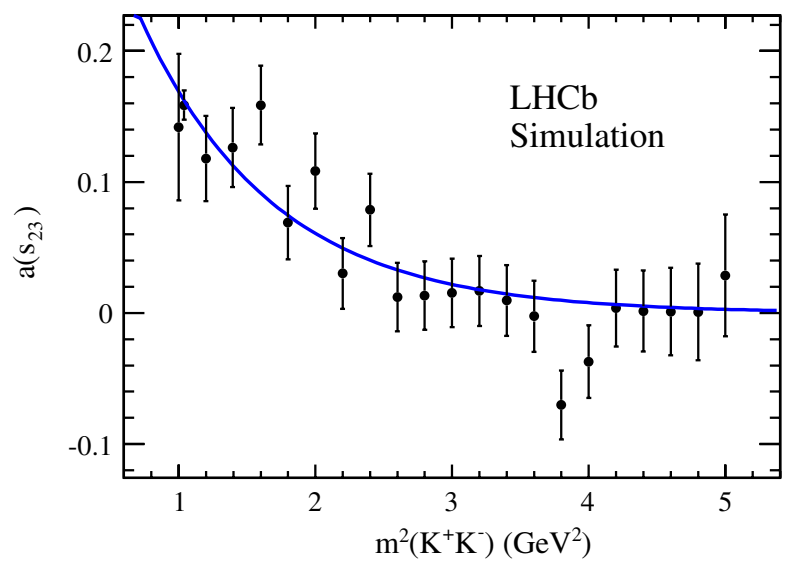

FIG. 7 (color online). Exponential fit to the efficiency parameter $a\left(s_{23}\right)$. The point near the $\phi(1020)$ meson mass is determined more precisely due to the use of a large simulation sample. 


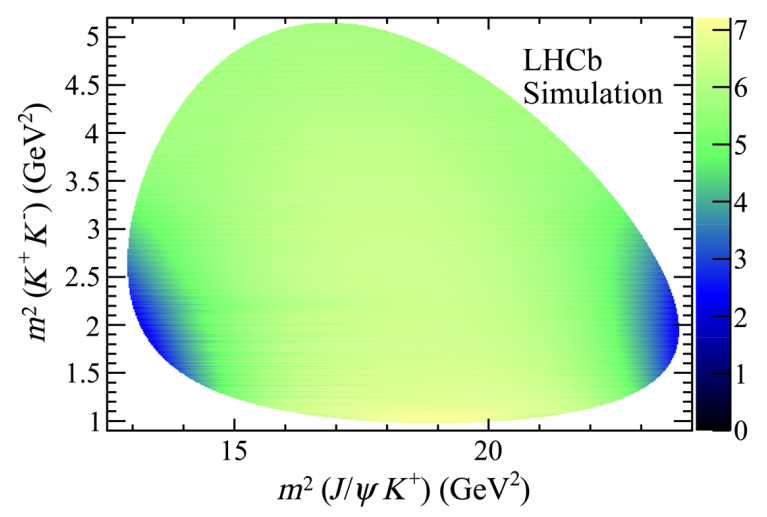

FIG. 8 (color online). Parametrized detection efficiency as a function of $m^{2}\left(K^{+} K^{-}\right)$versus $m^{2}\left(J / \psi K^{+}\right)$. The $z$-axis scale is arbitrary.

to be independent of $s_{12}$. Thus the detection efficiency can be expressed as

$$
\varepsilon\left(s_{12}, s_{23}, \theta_{J / \psi}\right)=\varepsilon_{1}(x, y) \times \varepsilon_{2}\left(s_{23}, \theta_{J / \psi}\right) .
$$

After integrating over $\cos \theta_{J / \psi}$, Eq. (20) becomes

$$
\int_{-1}^{+1} \varepsilon\left(s_{12}, s_{23}, \theta_{J / \psi}\right) d \cos \theta_{J / \psi}=\varepsilon_{1}(x, y)
$$

and is modeled by a symmetric fifth-order polynomial function given by

$$
\begin{aligned}
\varepsilon_{1}(x, y)= & 1+\epsilon_{1}^{\prime}(x+y)+\epsilon_{2}^{\prime}(x+y)^{2}+\epsilon_{3}^{\prime} x y \\
& +\epsilon_{4}^{\prime}(x+y)^{3}+\epsilon_{5}^{\prime} x y(x+y)+\epsilon_{6}^{\prime}(x+y)^{4} \\
& +\epsilon_{7}^{\prime} x y(x+y)^{2}+\epsilon_{8}^{\prime} x^{2} y^{2}+\epsilon_{9}^{\prime}(x+y)^{5} \\
& +\epsilon_{10}^{\prime} x y(x+y)^{3}+\epsilon_{11}^{\prime} x^{2} y^{2}(x+y),
\end{aligned}
$$

where $\epsilon_{i}^{\prime}$ are the fit parameters. The $\bar{B}_{s}^{0} \rightarrow J / \psi K^{+} K^{-}$ phase space simulation sample is modeled with the polynomial function. The fitted function is shown

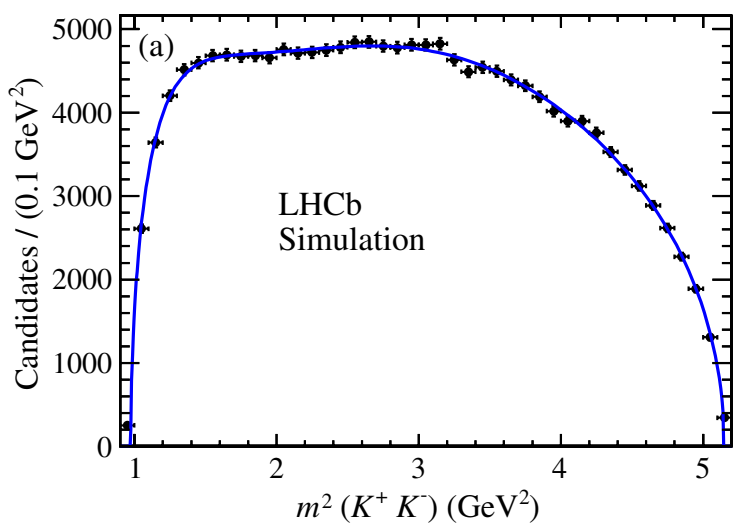

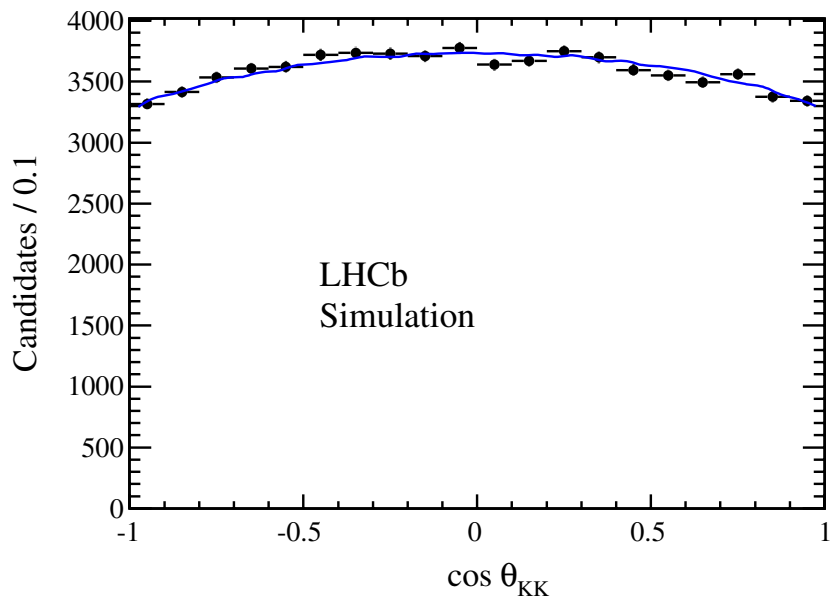

FIG. 10 (color online). Distribution of $\cos \theta_{K K}$ for the $J / \psi \phi$ simulated sample fitted with $\varepsilon_{1}(x, y) \times A\left(\theta_{K K}\right)$, within $\pm 20 \mathrm{MeV}$ of the $\phi(1020)$ mass.

in Fig. 8, and the projections of the fit are shown in Fig. 9. The efficiency is well described by the parametrization.

For the region within $\pm 20 \mathrm{MeV}$ of the $\phi(1020)$ mass, the $\cos \theta_{K K}$ acceptance is used separately, due to the large number of signal events. Here the $\cos \theta_{K K}$ distribution shows a variation in efficiency, which can be parametrized using the efficiency function

$$
A\left(\theta_{K K}\right)=\frac{1+\epsilon_{12}^{\prime} \cos ^{2} \theta_{K K}}{1+\epsilon_{12}^{\prime} / 3}
$$

where the parameter $\epsilon_{12}^{\prime}$ is measured from a fit to the simulated $J / \psi \phi$ sample with $\varepsilon_{1}(x, y) \times A\left(\theta_{K K}\right)$, giving $\epsilon_{12}^{\prime}=-0.099 \pm 0.010$, as shown in Fig. 10.

The mass resolution is $\sim 0.7 \mathrm{MeV}$ at the $\phi(1020)$ mass peak, which is added to the fit model by increasing the Breit-Wigner width of the $\phi(1020)$ to $4.59 \mathrm{MeV}$.

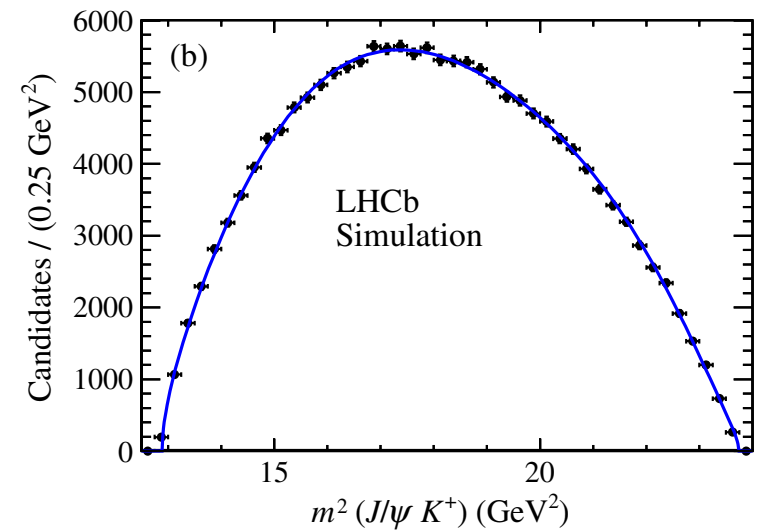

FIG. 9 (color online). Projections of the invariant mass squared (a) $K^{+} K^{-}$and (b) $J / \psi K^{+}$from the simulation used to measure the efficiency parameters. The points represent the generated event distributions and the curves the polynomial fit. 

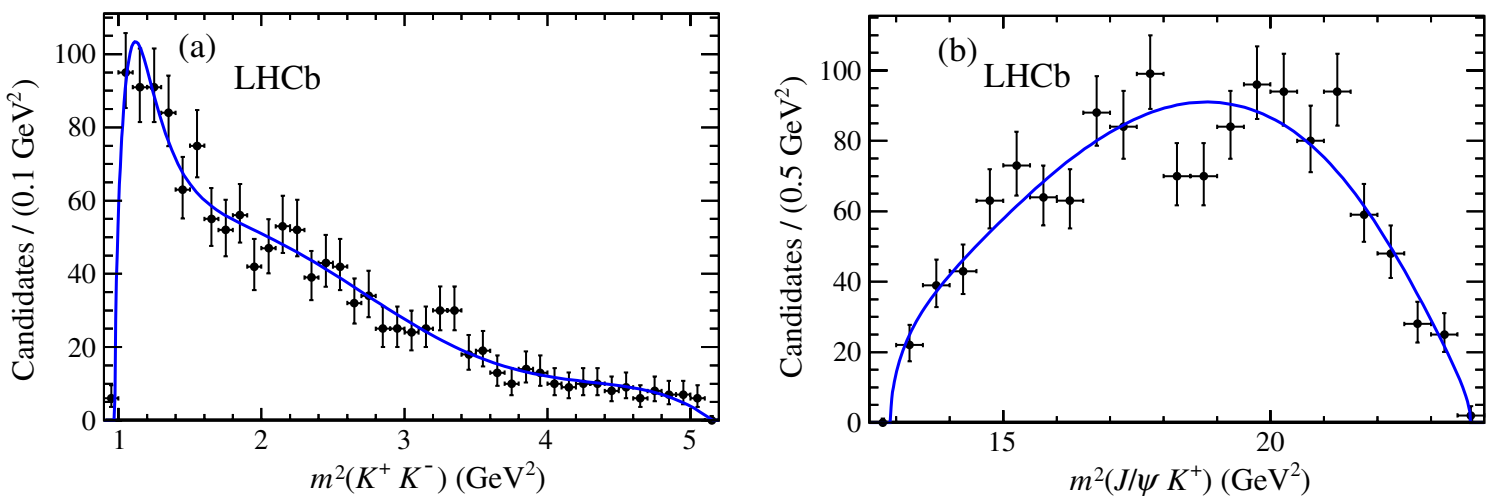

FIG. 11 (color online). Invariant mass squared projections of (a) $K^{+} K^{-}$and (b) $J / \psi K^{+}$from the background Dalitz plot of candidates in the $\bar{B}_{s}^{0}$ mass sidebands.

\section{Background composition}

The shape of the combinatorial background is modeled as

$$
\begin{aligned}
C\left(s_{12}, s_{23}, \theta_{J / \psi}\right)= & {\left[C_{1}\left(s_{12}, s_{23}\right) \frac{P_{B}}{m_{B}}+\frac{c_{0}}{\left(m_{0}^{2}-s_{23}\right)^{2}+m_{0}^{2} \Gamma_{0}^{2}}\right] } \\
& \times\left(1+\alpha \cos ^{2} \theta_{J / \psi}\right),
\end{aligned}
$$

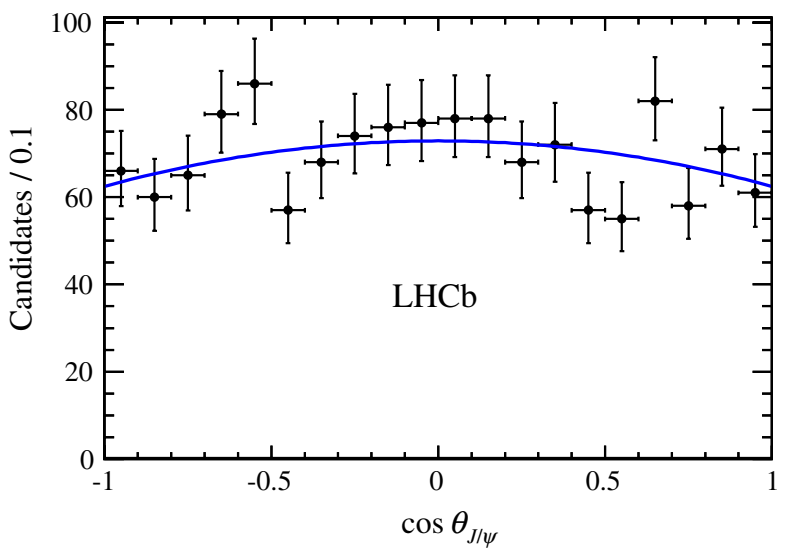

FIG. 12 (color online). Distribution of $\cos \theta_{J / \psi}$ from the background sample fit with the function $1+\alpha \cos ^{2} \theta_{J / \psi}$.

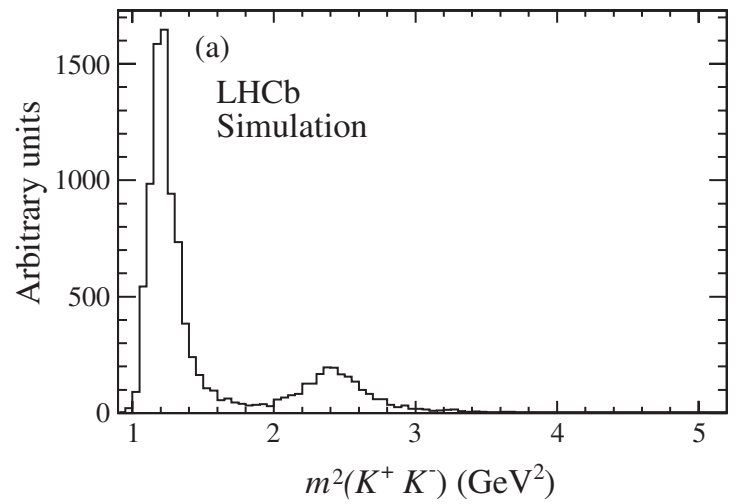

where $C_{1}\left(s_{12}, s_{23}\right)$ is parametrized as

$$
\begin{aligned}
C_{1}\left(s_{12}, s_{23}\right)= & 1+c_{1}(x+y)+c_{2}(x+y)^{2}+c_{3} x y \\
& +c_{4}(x+y)^{3}+c_{5} x y(x+y),
\end{aligned}
$$

with $c_{i}, m_{0}, \Gamma_{0}$ and $\alpha$ as the fit parameters. The variables $x$ and $y$ are defined in Eq. (16).

Figure 11 shows the mass squared projections from the $\bar{B}_{s}^{0}$ mass sidebands with the fit projections overlaid. The $\chi^{2} / \mathrm{ndf}$ of the fit is $291 / 305$. The value of $\alpha$ is determined by fitting the $\cos \theta_{J / \psi}$ distribution of background, as shown in Fig. 12, with a function of the form $1+\alpha \cos ^{2} \theta_{J / \psi}$, yielding $\alpha=-0.14 \pm 0.08$.

The reflection background is parametrized as

$$
R\left(s_{12}, s_{23}, \theta_{J / \psi}\right)=R_{1}\left(s_{12}, s_{23}\right) \times\left(1+\beta \cos ^{2} \theta_{J / \psi}\right),
$$

where $R_{1}\left(s_{12}, s_{23}\right)$ is modeled using the simulation; the projections of $s_{12}$ and $s_{23}$ are shown in Fig. 13. The $J / \psi$ helicity angle dependent part of the reflections is modeled as $1+\beta \cos ^{2} \theta_{J / \psi}$, where the parameter $\beta$ is obtained from a fit to the simulated $\cos \theta_{J / \psi}$ distribution, shown in Fig. 14, giving $\beta=-0.19 \pm 0.01$.

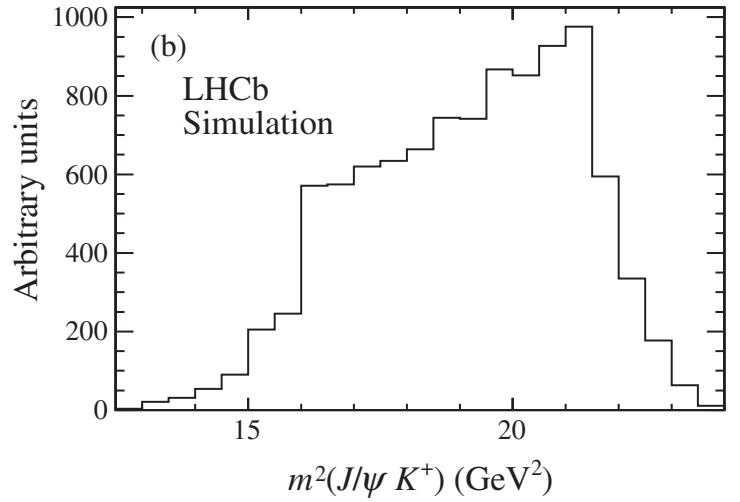

FIG. 13. Projections of the reflection background in the variables (a) $m^{2}\left(K^{+} K^{-}\right)$and (b) $m^{2}\left(J / \psi K^{+}\right)$, obtained from $\bar{B}^{0} \rightarrow J / \psi \bar{K}^{* 0}(892)$ and $\bar{B}^{0} \rightarrow J / \psi_{2} \bar{K}_{2}^{*}(1430)$ simulations. 


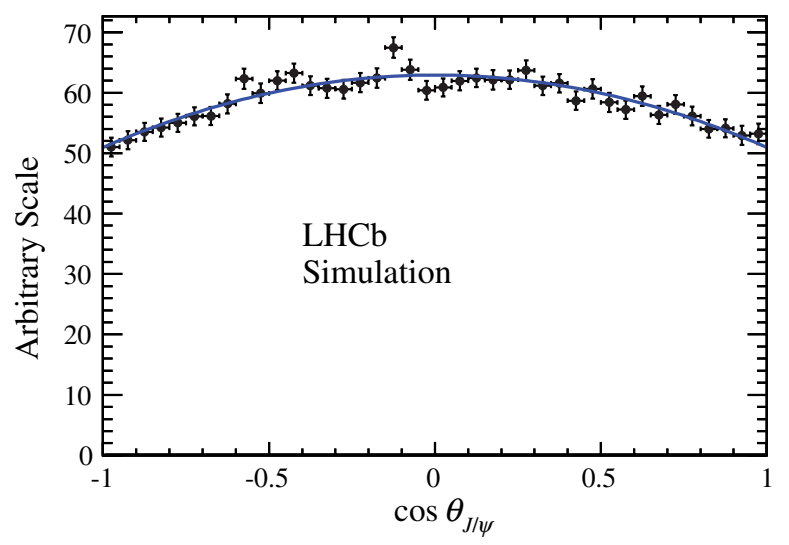

FIG. 14 (color online). Distribution of $\cos \theta_{J / \psi}$ for the reflection fit with the function $1+\beta \cos ^{2} \theta_{J / \psi}$.

\section{FINAL STATE COMPOSITION}

\section{A. Resonance models}

The resonances that are likely to contribute are produced from the $s \bar{s}$ system in Fig. 1, and thus are isoscalar $(I=0)$. The $K^{+} K^{-}$system in the decay $\bar{B}_{s}^{0} \rightarrow J / \psi K^{+} K^{-}$can, in principle, have zero or any positive integer angular momentum. Both the $P$-parity and $C$-parity of $K^{+} K^{-}$pair in a state of relative angular momentum $L$ are given by $(-1)^{L}$. Therefore the allowed resonances decaying to $K^{+} K^{-}$are limited to $J^{P C}=0^{++}, 1^{--}, 2^{++}, \ldots$, with isospin $I=0$. In the kinematically accessible mass range up to $2 \mathrm{GeV}$, resonances with $J^{P C}=3^{--}$or higher are not expected and thus the subsequent analysis only uses spins up to $J=2$. Possible resonance candidates are listed in Table I. There could also be a contribution from nonresonant events which we assume to be $S$-wave and evenly distributed over the available phase space.

To study the resonant structures of the decay $\bar{B}_{s}^{0} \rightarrow$ $J / \psi K^{+} K^{-}$we use 20,425 candidates with an invariant mass within $\pm 20 \mathrm{MeV}$ of the observed $\bar{B}_{s}^{0}$ mass peak. This includes both signal and background, with $94 \%$ signal purity. We begin our analysis considering only the resonance components $\phi(1020), f_{2}^{\prime}(1525)$ and a nonresonant component, established in our earlier measurement [10], and add resonances until no others are found with more than two standard deviation statistical significance $(2 \sigma)$. The significance is estimated from the fit fraction divided by its statistical uncertainty. Our best fit model includes a nonresonant component and eight resonance states: $\phi(1020), f_{0}(980), f_{0}(1370), f_{2}^{\prime}(1525), f_{2}(1640)(|\lambda|=$ $1), \phi(1680)(|\lambda|=1),{ }^{4} f_{2}(1750)$, and $f_{2}(1950)$. Most of the resonances considered here are well established except for the modes $f_{2}(1640), f_{2}(1750)$, and $f_{2}(1950)$. Although

\footnotetext{
${ }^{4}$ The $f_{2}(1640)(\lambda=0)$ and $\phi(1680)(\lambda=0)$ components have less than two standard deviation significance when added separately to the fit, and therefore are not included in the best fit model.
}

TABLE I. Possible resonance candidates in the $\bar{B}_{s}^{0} \rightarrow$ $J / \psi K^{+} K^{-}$decay mode.

\begin{tabular}{lccc}
\hline \hline Spin & Helicity & Resonance & Amplitude \\
\hline 0 & 0 & $f_{0}(980)$ & Flatté \\
0 & 0 & $f_{0}(1370), f_{0}(1500), f_{0}(1710)$ & BW \\
1 & $0, \pm 1$ & $\phi(1020), \phi(1680)$ & BW \\
2 & $0, \pm 1$ & $f_{2}(1270), f_{2}^{\prime}(1525), f_{2}(1640)$, & BW \\
& \multicolumn{4}{c}{$f_{2}(1750), f_{2}(1950)$} \\
\hline \hline
\end{tabular}

the existence of $f_{2}(1640)$ is not confirmed yet [9], the right shoulder of $f_{2}^{\prime}(1525)$ fits better when we add this state. The presence of multiple broad overlapping resonances in this region may indicate a failure of the isobar model used in this analysis, but with the present data sample alternative descriptions are not feasible. Indeed, the situation is not clear for the resonance states in the vicinity of $1750 \mathrm{MeV}$. The PDG lists a spin-0 resonance, $f_{0}(1710)$, around $1.72 \mathrm{GeV}$ of $\mathrm{K}^{+} K^{-}$invariant mass [9]. The Belle collaboration observed a resonance in the vicinity of $1.75 \mathrm{GeV}$ with $J^{P C}=(\text { even })^{++}$in their study of $\gamma \gamma \rightarrow K^{+} K^{-}$[28], but could not establish its spin. A state of mass $1767 \pm$ $14 \mathrm{MeV}$ was seen by the L3 collaboration decaying into $K_{S}^{0} K_{S}^{0}$ with $J=2$ [29]. We find that our data are better fit including the $f_{2}(1750)$ mode. If we substitute either the $f_{0}(1710)$ or $f_{0}(1750)$ resonance the fit is worsened, as the $-\ln \mathcal{L}$ increase by 59 and 7 units, respectively.

In the same analysis of $\gamma \gamma \rightarrow K^{+} K^{-}$, Belle also observed the $f_{2}(1950)$ [28] resonance. We include this state in our best fit model. Furthermore, we do not expect significant contributions from the $f_{2}(1270)$ and $f_{0}(1500)$ resonances, since the PDG branching fractions are much larger in the $\pi^{+} \pi^{-}$final state than in $K^{+} K^{-}$[9] and we did not see significant contributions from these two resonances in the $\bar{B}_{s}^{0} \rightarrow J / \psi \pi^{+} \pi^{-}$final state [8]. Therefore, these two resonances are not considered in the best fit model. However, we add these states, in turn, to the best fit model in order to test for their possible presence.

The masses and widths of the BW resonances are listed in Table II. When used in the fit they are fixed to the central values, except for the $f_{2}^{\prime}(1525)$, whose mass and width are allowed to vary.

The $f_{0}(980)$ is described by a Flatté resonance shape, see Eq. (12). The parameters describing the function are the mass, and the couplings $g_{\pi \pi}$ and $g_{K K}$, which are fixed in the fit from the previous analysis of $\bar{B}_{s}^{0} \rightarrow J / \psi \pi^{+} \pi^{-}$ [8]. The parameters are $m_{0}=939.9 \pm 6.3 \mathrm{MeV}, g_{\pi \pi}=$ $199 \pm 30 \mathrm{MeV}$ and $g_{K K} / g_{\pi \pi}=3.0 \pm 0.3$. All background and efficiency parameters are fixed in the fit.

To determine the complex amplitudes in a specific model, the data are fitted maximizing the unbinned likelihood given as

$$
\mathcal{L}=\prod_{i=1}^{N} F\left(s_{12}^{i}, s_{23}^{i}, \theta_{J / \psi}^{i}\right),
$$


TABLE II. Breit-Wigner resonance parameters.

\begin{tabular}{lccc}
\hline \hline Resonance & Mass $(\mathrm{MeV})$ & Width $(\mathrm{MeV})$ & Source \\
\hline$\phi(1020)$ & $1019.46 \pm 0.02$ & $4.26 \pm 0.04$ & PDG [9] \\
$f_{2}(1270)$ & $1275 \pm 1$ & $185 \pm 3$ & PDG [9] \\
$f_{0}(1370)$ & $1475 \pm 6$ & $113 \pm 11$ & LHCb [8] \\
$f_{0}(1500)$ & $1505 \pm 6$ & $109 \pm 7$ & PDG [9] \\
$f_{2}^{\prime}(1525)$ & $1525 \pm 5$ & $73 \pm 6$ & PDG [9] \\
$f_{2}(1640)$ & $1639 \pm 6$ & $99 \pm 60$ & PDG [9] \\
$\phi(1680)$ & $1680 \pm 20$ & $150 \pm 50$ & PDG [9] \\
$f_{0}(1710)$ & $1720 \pm 6$ & $135 \pm 8$ & PDG [9] \\
$f_{2}(1750)$ & $1737 \pm 9$ & $151 \pm 33$ & Belle [28] \\
$f_{2}(1950)$ & $1980 \pm 14$ & $297 \pm 13$ & Belle [28] \\
\hline \hline
\end{tabular}

where $N$ is the total number of candidates, and $F$ is the total PDF defined in Eq. (1). The PDF normalization is accomplished by first normalizing the $J / \psi$ helicity dependent part by analytical integration, and then for the mass dependent part using numerical integration over $400 \times 800$ bins.

The fit determines the relative values of the amplitude strengths, $a_{\lambda}^{R_{i}}$, and phases, $\phi_{\lambda}^{R_{i}}$, defined in Eq. (4). We choose to fix $a_{0}^{\phi(1020)}=1$. As only relative phases are physically meaningful, one phase in each helicity grouping must be fixed. In addition, because $J / \psi K^{+} K^{-}$is a self-charge-conjugate mode and does not determine the initial $B$ flavor, the signal function is an average of $B_{s}^{0}$ and $\bar{B}_{s}^{0}$. If we consider no $K^{+} K^{-}$partial-waves of a higher order than $D$-wave, then we can express the differential decay rate $\left(d \Gamma / d m_{K K} d \cos \theta_{K K} d \cos \theta_{J / \psi}\right)$ derived from Eq. (4) in terms of $S-, P$-, and $D$-waves including helicity 0 and \pm 1 components. The differential decay rates for $\bar{B}_{s}^{0}$ and $B_{s}^{0}$, respectively are

$$
\begin{aligned}
& \frac{d \bar{\Gamma}}{d m_{K K} d \cos \theta_{K K} d \cos \theta_{J / \psi}}=\left|\mathcal{A}_{S_{0}}^{s} e^{i \phi_{S_{0}}^{s}}+\mathcal{A}_{P_{0}}^{s} e^{i \phi_{P_{0}}^{s}} \cos \theta_{K K}+\mathcal{A}_{D_{0}}^{s} e^{i \phi_{D_{0}}^{s}}\left(\frac{3}{2} \cos ^{2} \theta_{K K}-\frac{1}{2}\right)\right|^{2} \sin ^{2} \theta_{J / \psi} \\
& +\left|\mathcal{A}_{P_{ \pm 1}}^{s} e^{i \phi_{P_{ \pm 1}}^{s}} \sqrt{\frac{1}{2}} \sin \theta_{K K}+\mathcal{A}_{D_{ \pm 1}}^{s} e^{i \phi_{D_{ \pm}}^{s}} \sqrt{\frac{3}{2}} \sin \theta_{K K} \cos \theta_{K K}\right| \frac{21+\cos ^{2} \theta_{J} / \psi}{2},
\end{aligned}
$$

and

$$
\begin{aligned}
\frac{d \Gamma}{d m_{K K} d \cos \theta_{K K} d \cos \theta_{J / \psi}}= & \left|\mathcal{A}_{S_{0}}^{s} e^{i \phi_{S_{0}}^{s}}-\mathcal{A}_{P_{0}}^{s} e^{i \phi_{P_{0}}^{s}} \cos \theta_{K K}+\mathcal{A}_{D_{0}}^{s} e^{i \phi_{D_{0}}^{s}}\left(\frac{3}{2} \cos ^{2} \theta_{K K}-\frac{1}{2}\right)\right|^{2} \sin ^{2} \theta_{J / \psi} \\
& +\left|\mathcal{A}_{P_{ \pm 1}}^{s} e^{i \phi_{P_{ \pm 1}}^{s}} \sqrt{\frac{1}{2}} \sin \theta_{K K}-\mathcal{A}_{D_{ \pm 1}}^{s} e^{i \phi_{D_{ \pm}}^{s}} \sqrt{\frac{3}{2}} \sin \theta_{K K} \cos \theta_{K K}\right| \frac{21+\cos ^{2} \theta_{J / \psi}}{2},
\end{aligned}
$$

where $\mathcal{A}_{k_{\lambda}}^{s}$ and $\phi_{k_{\lambda}}^{s}$ are the sum of amplitudes and reference phases, for the spin- $k$ resonance group, respectively. The decay rate for $B_{s}^{0}$ is similar to that of $\bar{B}_{s}^{0}$, except $\theta_{K^{+} K^{-}}$ and $\theta_{J / \psi}$ are now changed to $\pi-\theta_{K^{+} K^{-}}$and $\pi-\theta_{J / \psi}$ respectively, as a result of using $K^{-}$and $\mu^{-}$to define the helicity angles and hence the signs change in front of the $\mathcal{A}_{P_{0}}^{s}$ and $\mathcal{A}_{D_{+1}}^{s}$ terms.

Summing Eqs. (28) and (29) results in cancellation of the interference involving $\lambda=0$ terms for spin- 1 , and the $\lambda= \pm 1$ terms for spin-2, as they appear with opposite signs for $\bar{B}_{s}^{0}$ and $B_{s}^{0}$ decays. Therefore we have to fix one phase in the spin-1 $(\lambda=0)$ group $\left(\phi_{P_{0}}^{s}\right)$ and one in the spin-2 $(\lambda= \pm 1)$ group $\left(\phi_{D_{ \pm 1}}^{s}\right)$. The other phases in each corresponding group are determined relative to that of the fixed resonance.

\section{B. Fit results}

The goodness of fit is calculated from 3D partitions of $s_{12}, s_{23}$ and $\cos \theta_{J / \psi}$. We use the Poisson likelihood $\chi^{2}$ [30] defined as

$$
\chi^{2}=2 \sum_{i=1}^{N}\left[x_{i}-n_{i}+n_{i} \ln \left(\frac{n_{i}}{x_{i}}\right)\right],
$$

where $n_{i}$ is the number of candidates in the threedimensional bin $i$ and $x_{i}$ is the expected number of candidates in that bin according to the fitted likelihood function. An adaptive binning algorithm is used, requiring a minimum of 25 entries in each bin. The associated number of degrees of freedom is $N-k-1$, where $k$ is the number of free parameters in the likelihood function. The $\chi^{2} / \mathrm{ndf}$ and the negative of the logarithm of the likelihood, $-\ln \mathcal{L}$, of the fits are given in Table III. Starting values of parameters are varied in order to ensure that global likelihood minimums are found rather than local minimums.

Attempts to add one more resonance such as $f_{2}(1270)$ and $f_{0}(1500)$ improve the $-\ln \mathcal{L}$ marginally, but the $\chi^{2} /$ ndf are worse than the best fit model. We retain only those resonances that are more than $2 \sigma$ significant, except

TABLE III. $\quad \chi^{2} /$ ndf and $-\ln \mathcal{L}$ of different resonance models.

\begin{tabular}{lcc}
\hline \hline Resonance model & $-\ln \mathcal{L}$ & $\chi^{2} /$ ndf \\
\hline Best fit & 29,275 & $649 / 545=1.1908$ \\
Best fit $+f_{2}(1270)$ & 29,273 & $644 / 541=1.1911$ \\
Best fit $+f_{0}(1500)$ & 29,274 & $647 / 543=1.1915$ \\
\hline \hline
\end{tabular}


for the $f_{2}(1750)$ where we allow the $|\lambda|=1$ component since the $\lambda=0$ component is significant. For models with one more resonance, the additional components never have more than $2 \sigma$ significance. Figure 15 shows the projection of $m^{2}\left(K^{+} K^{-}\right)$for the best fit model, the $m^{2}\left(J / \psi K^{+}\right)$and $\cos \theta_{J / \psi}$ projections are displayed in Fig. 16. The projection of the $K^{+} K^{-}$invariant mass spectrum is shown in Fig. 17.

While a complete description of the $\bar{B}_{s}^{0} \rightarrow J / \psi K^{+} K^{-}$ decay is given in terms of the fitted amplitudes and phases, knowledge of the contribution of each component can be summarized by defining a fit fraction, $\mathcal{F}_{\lambda}^{R}$. To determine
$\mathcal{F}_{\lambda}^{R}$ we integrate the squared amplitude of $R$ over the Dalitz plot. The yield is then normalized by integrating the entire signal function over the same area. Specifically,

$\mathcal{F}_{\lambda}^{R}=\frac{\int\left|a_{\lambda}^{R} e^{i \phi_{\lambda}^{R}} \mathcal{A}_{\lambda}^{R}\left(s_{12}, s_{23}, \theta_{J / \psi}\right)\right|^{2} d s_{12} d s_{23} d \cos \theta_{J / \psi}}{\int S\left(s_{12}, s_{23}, \theta_{J / \psi}\right) d s_{12} d s_{23} d \cos \theta_{J / \psi}}$.

Note that the sum of the fit fractions is not necessarily unity due to the potential presence of interference between two resonances. Interference term fractions are given by

$$
\mathcal{F}_{\lambda}^{R R^{\prime}}=\operatorname{Re}\left(\frac{\int a_{\lambda}^{R} a_{\lambda}^{R^{\prime}} e^{i\left(\phi_{\lambda}^{R}-\phi_{\lambda}^{R^{\prime}}\right)} \mathcal{A}_{\lambda}^{R}\left(s_{12}, s_{23}, \theta_{J / \psi}\right) \mathcal{A}_{\lambda}^{R^{\prime *}}\left(s_{12}, s_{23}, \theta_{J / \psi}\right) d s_{12} d s_{23} d \cos \theta_{J / \psi}}{\int S\left(s_{12}, s_{23}, \theta_{J / \psi}\right) d s_{12} d s_{23} d \cos \theta_{J / \psi}}\right),
$$

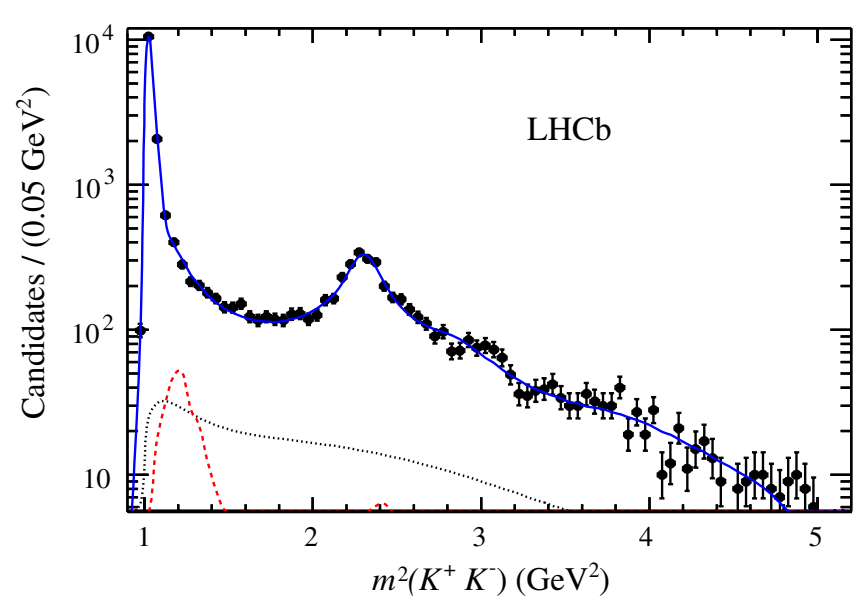

FIG. 15 (color online). Dalitz plot fit projection of $m^{2}\left(K^{+} K^{-}\right)$ using a logarithmic scale. The points with error bars are data, the dotted curve (black) shows the combinatorial background, the dashed curve (red) indicates the reflection from the misidentified $\bar{B}^{0} \rightarrow$ $J / \psi K^{-} \pi^{+}$decays, and the solid line (blue) represents the total. and

$$
\sum_{\lambda}\left(\sum_{R} \mathcal{F}_{\lambda}^{R}+\sum_{R R^{\prime}}^{R \neq R^{\prime}} \mathcal{F}_{\lambda}^{R R^{\prime}}\right)=1 .
$$

If the Dalitz plot has more destructive interference than constructive interference, the sum of the fit fractions will be greater than unity. Conversely, the sum will be less than one if the Dalitz plot exhibits constructive interference. Note that interference between different spin- $J$ states vanishes because the $d_{\lambda 0}^{J}$ angular functions in $\mathcal{A}_{\lambda}^{R}$ are orthogonal.

The determination of the statistical uncertainties of the fit fractions is difficult because they depend on the statistical uncertainty of every fitted magnitude and phase. Therefore we determine the uncertainties from simulated experiments. We perform 500 experiments: each sample is generated according to the model PDF, input parameters are taken from the fit to the data. The correlations of fitted parameters are also taken into account. For each
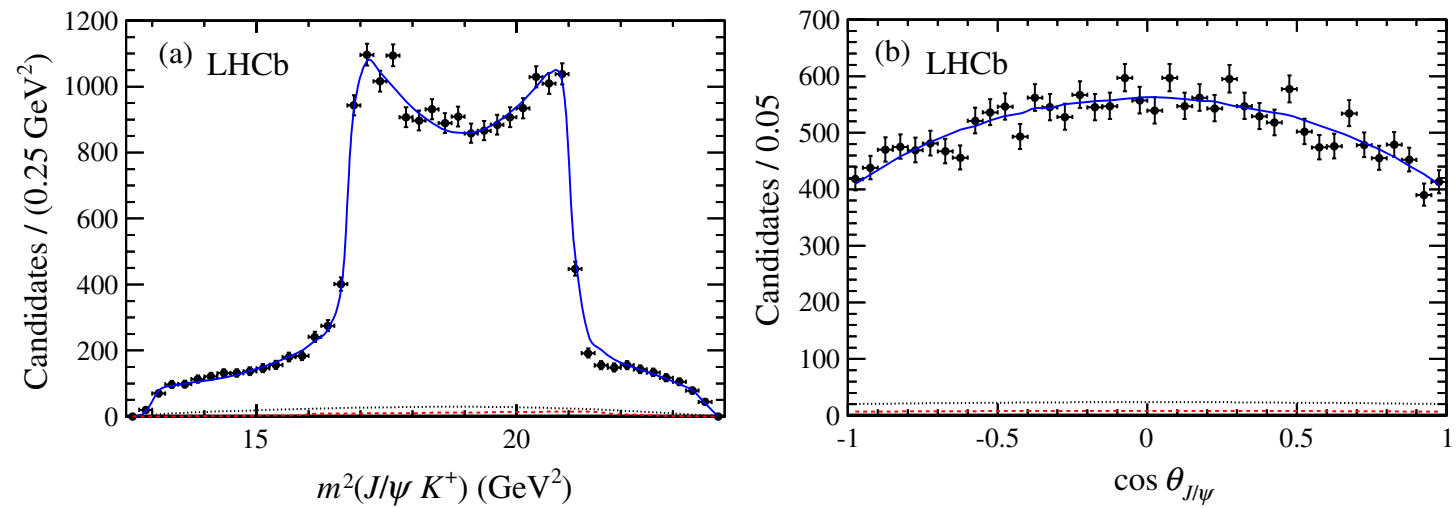

FIG. 16 (color online). Dalitz plot fit projections of (a) $m^{2}\left(J / \psi K^{+}\right)$and (b) $\cos \theta_{J / \psi}$. The points with error bars are data, the (black) dotted curve shows the combinatorial background, the (red) dashed curve indicates the reflection from the misidentified $\bar{B}^{0} \rightarrow$ $J / \psi K^{-} \pi^{+}$decays, and the (blue) solid line represents the total fit results. 


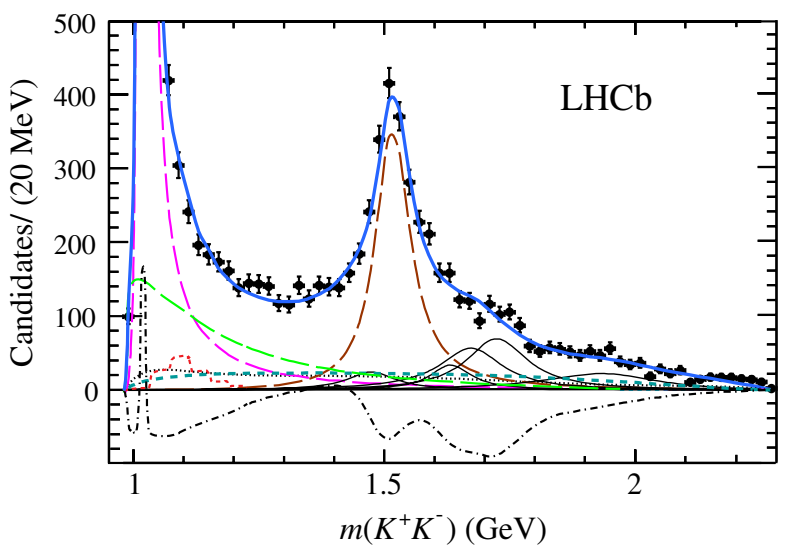

FIG. 17 (color online). Dalitz fit projection of $m\left(K^{+} K^{-}\right)$. The points represent the data, the (black) dotted curve shows the combinatorial background, and the (red) dashed curve indicates the reflection from misidentified $\bar{B}^{0} \rightarrow J / \psi K^{-} \pi^{+}$decays. The largest three resonances $\phi(1020), f_{2}^{\prime}(1525)$ and $f_{0}(980)$ are shown by magenta, brown and green long-dashed curves, respectively; all other resonances are shown by (black) thin curves. The (cyan) dashed curve is the nonresonant contribution. The (black) dot-dashed curve is the contribution from the interferences, and the (blue) solid curve represents the total fit result.

experiment the fit fractions are calculated. The distributions of the obtained fit fractions are described by Gaussian functions. The r.m.s. widths of the Gaussian functions are taken as the statistical uncertainties on the corresponding parameters. The fit fractions and phases of the contributing components are given in Table IV, while the fit fractions of the interference terms are quoted in Table V.

TABLE IV. Fit fractions (\%) and phases of contributing components. For $P$ - and $D$-waves $\lambda$ represents the helicity.

\begin{tabular}{lcc}
\hline \hline Component & Fit fraction $(\%)$ & Phase (degree) \\
\hline$\phi(1020), \lambda=0$ & $32.1 \pm 0.5 \pm 0.8$ & 0 (fixed) \\
$\phi(1020),|\lambda|=1$ & $34.6 \pm 0.5 \pm 1.3$ & 0 (fixed) \\
$f_{0}(980)$ & $12.0 \pm 1.8_{-2.5}^{+2.8}$ & $-294 \pm 8 \pm 25$ \\
$f_{0}(1370)$ & $1.2 \pm 0.3_{-1.2}^{+0.3}$ & $-81 \pm 8 \pm 8$ \\
$f_{2}^{\prime}(1525), \lambda=0$ & $9.9 \pm 0.7_{-1.6}^{+2.4}$ & 0 (fixed) \\
$f_{2}^{\prime}(1525),|\lambda|=1$ & $5.1 \pm 0.9_{-1.4}^{+1.8}$ & $0($ fixed $)$ \\
$f_{2}(1640),|\lambda|=1$ & $1.5 \pm 0.7_{-0.9}^{+0.7}$ & $165 \pm 27_{-44}^{+13}$ \\
$\phi(1680),|\lambda|=1$ & $3.4 \pm 0.3_{-0.3}^{+4.4}$ & $106 \pm 14_{-210}^{+260}$ \\
$f_{2}(1750), \lambda=0$ & $2.6 \pm 0.5_{-0.6}^{+1.0}$ & $238 \pm 8 \pm 9$ \\
$f_{2}(1750),|\lambda|=1$ & $1.8 \pm 1.0_{-1.8}^{+2.2}$ & $45 \pm 30_{-70}^{+16}$ \\
$f_{2}(1950), \lambda=0$ & $0.4 \pm 0.2_{-0.4}^{+0.2}$ & $46 \pm 17_{-20}^{+110}$ \\
$f_{2}(1950),|\lambda|=1$ & $1.7 \pm 0.5_{-1.7}^{+2.5}$ & $-53 \pm 26_{-80}^{+150}$ \\
Nonresonant & $6.0 \pm 1.6_{-2.2}^{+2.0}$ & $-39 \pm 6 \pm 23$ \\
Total & 112.3 & \\
\hline \hline
\end{tabular}

Table VI shows a comparison of the fit fractions when a different parametrization is used for the $f_{0}(980)$ resonance shape. The BES $f_{0}(980)$ functional form is the same as ours with the parameters $m_{0}=965 \pm 10 \mathrm{MeV}, g_{\pi \pi}=$ $165 \pm 18 \mathrm{MeV}$ and $g_{K K} / g_{\pi \pi}=4.21 \pm 0.33$ [31]. The $B A B A R$ collaboration assumes that the nonresonant $S$-wave is small and consistent with zero [32]. The $B A B A R$ functional form is different and parametrized as

$$
A_{R}\left(s_{23}\right)=\frac{1}{m_{R}^{2}-s_{23}-i m_{R} \Gamma_{R} \rho_{K K}},
$$

with $\rho_{K K}=2 P_{R} / \sqrt{s_{23}}$. The parameters are $m_{R}=922 \pm$ $3 \mathrm{MeV}$ and $\Gamma_{R}=240 \pm 80 \mathrm{MeV}$, taken from BABAR's Dalitz plot analysis of $D_{s}^{+} \rightarrow K^{+} K^{-} \pi^{+}$[33]. The $f_{0}(980)$ fraction is smaller in the $B A B A R$ parametrization, while the total $S$-wave fraction is consistent in the three different parameterizations. In all cases, the dominant component is the $\phi(1020)$, the second largest contribution is the $f_{2}^{\prime}(1525)$, and the third the $f_{0}(980)$ resonance. There are also significant contributions from the $f_{0}(1370)$, $f_{2}(1640), \phi(1680), f_{2}(1750), f_{2}(1950)$ resonances, and nonresonant final states. The amount of $f_{0}(980)$ is strongly parametrization dependent, so we treat these three models separately and do not assign any systematic uncertainty based on the use of these different $f_{0}(980)$ shapes. Therefore we refrain from quoting a branching fraction measurement for the decay $\bar{B}_{s}^{0} \rightarrow J / \psi f_{0}(980)$.

The determination of the parameters of the $f_{2}^{\prime}(1525)$ resonance are not dependent on the $f_{0}(980)$ parametrization. The parameters of the $f_{2}^{\prime}(1525)$ are determined to be

$$
\begin{aligned}
m_{f_{2}^{\prime}(1525)} & =1522.2 \pm 2.8_{-2.0}^{+5.3} \mathrm{MeV} \\
\Gamma_{f_{2}^{\prime}(1525)} & =84 \pm 6_{-5}^{+10} \mathrm{MeV} .
\end{aligned}
$$

Whenever two or more uncertainties are quoted, the first is the statistical and the second systematic. The latter will be discussed in Section VF. These values are the most accurate determinations of the $f_{2}^{\prime}(1525)$ resonant parameters [9]. Note that our determination of the mass has the same uncertainty as the current PDG average.

\section{C. $K^{+} K^{-} S$-wave in the $\phi(1020)$ mass region}

It was claimed by Stone and Zhang [12] that in the decay of $\bar{B}_{s}^{0} \rightarrow J / \psi \phi$, the $K^{+} K^{-}$system can have $S$-wave contributions under the $\phi(1020)$ peak of order $7 \%$ of the total yield. In order to investigate this possibility we calculate the $S$-wave fractions as given by the fit in $4 \mathrm{MeV}$ mass intervals between $990<m\left(K^{+} K^{-}\right)<1050 \mathrm{MeV}$. The resulting behavior is shown in Fig. 18. Here we show the result from our preferred model and also from the alternative $f_{0}(980)$ parametrizations discussed above. The observation of significant $S$-wave fractions in this region means that this contribution must be taken into account when measuring $C P$ violation in the $\phi$ mass region. The total $S$-wave fraction 
TABLE V. Fit fractions matrix for the best fit in units of $\%$. The diagonal elements correspond to the decay fractions in Table IV. The off-diagonal elements give the fit fractions of the interference. The null values originate from the fact that any interference contribution between different spin- $J$ state integrates to zero. Here the resonances are labeled by their masses in $\mathrm{MeV}$ and the subscripts denote the helicities.

\begin{tabular}{|c|c|c|c|c|c|c|c|c|c|c|c|c|c|}
\hline & $1020_{0}$ & $1020_{1}$ & 980 & 1370 & $1525_{0}$ & $1525_{1}$ & $1640_{1}$ & $1680_{1}$ & $1750_{0}$ & $1750_{1}$ & $1950_{0}$ & $1950_{1}$ & $\mathrm{NR}$ \\
\hline $1020_{0}$ & 32.1 & 0 & 0 & 0 & 0 & 0 & 0 & 0 & 0 & 0 & 0 & 0 & 0 \\
\hline $1020_{1}$ & & 34.6 & 0 & 0 & 0 & 0 & 0 & 2.0 & 0 & 0 & 0 & 0 & 0 \\
\hline 980 & & & 12.0 & -2.3 & 0 & 0 & 0 & 0 & 0 & 0 & 0 & 0 & -4.7 \\
\hline 1370 & & & & 1.2 & 0 & 0 & 0 & 0 & 0 & 0 & 0 & 0 & 1.5 \\
\hline $1525_{0}$ & & & & & 9.9 & 0 & 0 & 0 & -4.5 & 0 & 0.9 & 0 & 0 \\
\hline $1525_{1}$ & & & & & & 5.1 & -0.9 & 0 & 0 & 2.5 & 0 & -1.8 & 0 \\
\hline $1640_{1}$ & & & & & & & 1.5 & 0 & 0 & -2.4 & 0 & 0.7 & 0 \\
\hline $1680_{1}$ & & & & & & & & 3.4 & 0 & 0 & 0 & 0 & 0 \\
\hline $1750_{0}$ & & & & & & & & & 2.6 & 0 & -0.4 & 0 & 0 \\
\hline $1750_{1}$ & & & & & & & & & & 1.8 & 0 & -2.2 & 0 \\
\hline $1950_{0}$ & & & & & & & & & & & 0.4 & 0 & 0 \\
\hline $1950_{1}$ & & & & & & & & & & & & 1.7 & 0 \\
\hline NR & & & & & & & & & & & & & 6.0 \\
\hline
\end{tabular}

TABLE VI. Comparison of the fit fractions (\%) with the LHCb, BES and BABAR $f_{0}(980)$ parametrizations described in the text. For $P$ - and $D$-waves, $\lambda$ represents the helicity.

\begin{tabular}{lccr}
\hline \hline Component & LHCb & BES & BABAR \\
\hline$\phi(1020), \lambda=0$ & $32.1 \pm 0.5$ & $32.1 \pm 0.5$ & $32.0 \pm 0.5$ \\
$\phi(1020),|\lambda|=1$ & $34.6 \pm 0.5$ & $34.6 \pm 0.5$ & $34.5 \pm 0.5$ \\
$f_{0}(980)$ & $12.0 \pm 1.8$ & $9.2 \pm 1.4$ & $4.8 \pm 1.0$ \\
$f_{0}(1370)$ & $1.2 \pm 0.3$ & $1.2 \pm 0.3$ & $1.3 \pm 0.3$ \\
$f_{2}^{\prime}(1525), \lambda=0$ & $9.9 \pm 0.7$ & $9.8 \pm 0.7$ & $9.5 \pm 0.7$ \\
$f_{2}^{\prime}(1525),|\lambda|=1$ & $5.1 \pm 0.9$ & $5.1 \pm 0.9$ & $4.9 \pm 0.9$ \\
$f_{2}(1640),|\lambda|=1$ & $1.5 \pm 0.7$ & $1.5 \pm 0.7$ & $1.5 \pm 0.7$ \\
$\phi(1680),|\lambda|=1$ & $3.4 \pm 0.3$ & $3.4 \pm 0.3$ & $3.4 \pm 0.3$ \\
$f_{2}(1750), \lambda=0$ & $2.6 \pm 0.5$ & $2.5 \pm 0.5$ & $2.2 \pm 0.5$ \\
$f_{2}(1750),|\lambda|=1$ & $1.8 \pm 1.0$ & $1.8 \pm 1.0$ & $1.9 \pm 1.0$ \\
$f_{2}(1950), \lambda=0$ & $0.4 \pm 0.2$ & $0.4 \pm 0.2$ & $0.4 \pm 0.2$ \\
$f_{2}(1950),|\lambda|=1$ & $1.7 \pm 0.5$ & $1.8 \pm 0.5$ & $1.8 \pm 0.5$ \\
Nonresonant $S$-wave & $6.0 \pm 1.4$ & $4.7 \pm 1.2$ & $8.6 \pm 1.7$ \\
Interference between $S$-waves & -5.5 & -1.7 & -1.1 \\
Total $S$-wave & 13.7 & 13.4 & 13.6 \\
$-\ln \mathcal{L}$ & 29,275 & 29,275 & 29,281 \\
$\chi^{2} /$ ndf & $649 / 545$ & $653 / 545$ & $646 / 545$ \\
\hline \hline
\end{tabular}

as a function of the mass interval around the $\phi$ mass is also shown in Fig. 19. Using a time dependent analysis of $\bar{B}_{s}^{0} \rightarrow$ $J / \psi \phi(1020), \mathrm{LHCb}$ reported $(2.2 \pm 1.2 \pm 0.07) \%$ [3] of $S$-wave within $\pm 12 \mathrm{MeV}$ of the $\phi(1020)$ mass peak. We measure the $S$-wave fraction within the same mass window as a consistent, and more precise $\left(1.1 \pm 0.1_{-0.1}^{+0.2}\right) \%$. CDF measured the $S$-wave fraction as $(0.8 \pm 0.2) \%$ for $m\left(K^{+} K^{-}\right)$within about $\pm 9.5 \mathrm{MeV}$ of the $\phi$ mass [6], while ATLAS quotes $(2 \pm 2) \%$ for an $11 \mathrm{MeV}$ interval [7]. These results are consistent with ours. The D0 collaboration, however, claimed a $(14.7 \pm 3.5) \% S$-wave fraction within approximately $\pm 10 \mathrm{MeV}$ of the $\phi$ meson mass [5], in disagreement with all of the other results.

\section{Helicity angle distributions}

The decay angular distributions or the helicity angle distributions are already included in the signal model via Eqs. (8) and (9). In order to test the fit model we examine the $\cos \theta_{J / \psi}$ and $\cos \theta_{K K}$ distributions in two different $K^{+} K^{-}$mass regions: one is the $\phi(1020)$ region defined within $\pm 12 \mathrm{MeV}$ of the $\phi(1020)$ mass peak and the other 


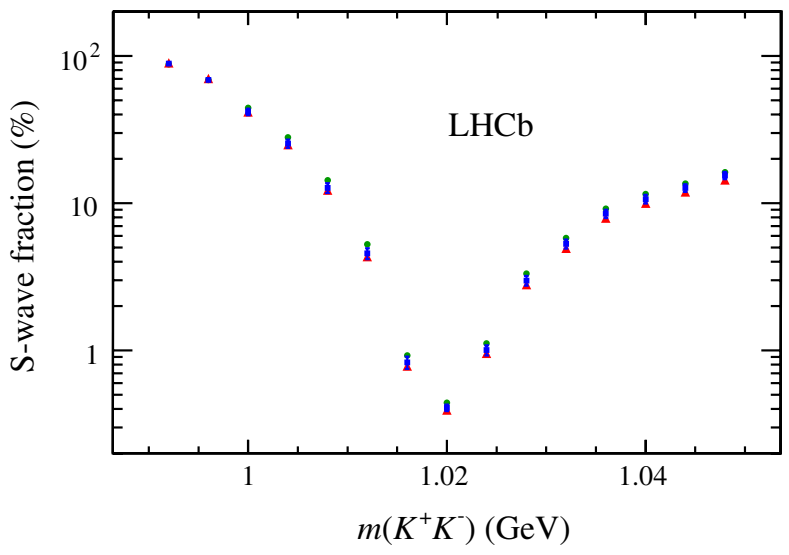

FIG. 18 (color online). $S$-wave fraction as a function of $m\left(K^{+} K^{-}\right)$starting from $990 \mathrm{MeV}$ up to $1050 \mathrm{MeV}$ in $4 \mathrm{MeV}$ mass intervals. The squares (blue), triangles (red), and circles (green) represent the $\mathrm{LHCb}, \mathrm{BES}$ and $B A B A R$ parametrizations of $f_{0}(980)$, respectively. The experimental statistical uncertainties are only shown for the LHCb model; they are almost identical for the other cases. The experimental mass resolution is not unfolded.

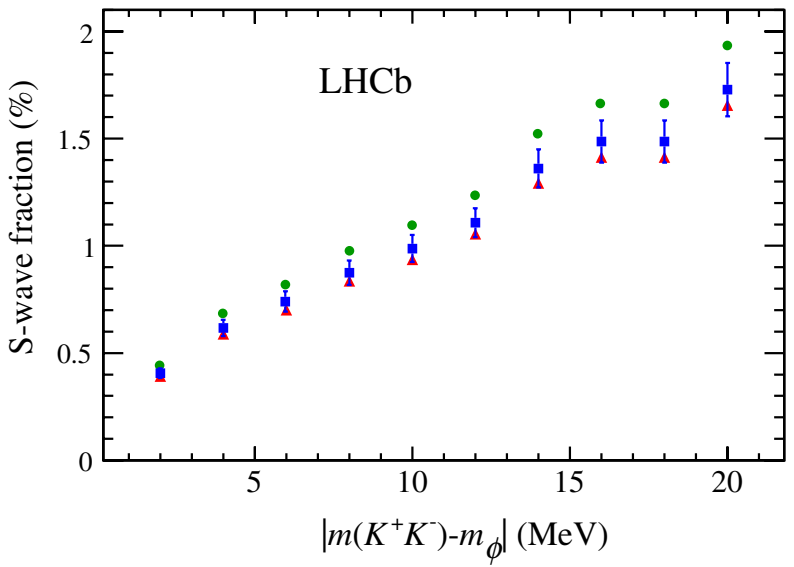

FIG. 19 (color online). $S$-wave fractions in different $m\left(K^{+} K^{-}\right)$intervals centered on the $\phi$ meson mass. The squares (blue), triangles (red), and circles (green) represent the LHCb, BES and $B A B A R$ parametrizations of $f_{0}(980)$, respectively. The experimental statistical uncertainties are only shown for the $\mathrm{LHCb}$ model; they are almost identical for the other cases. The experimental mass resolution is not unfolded.

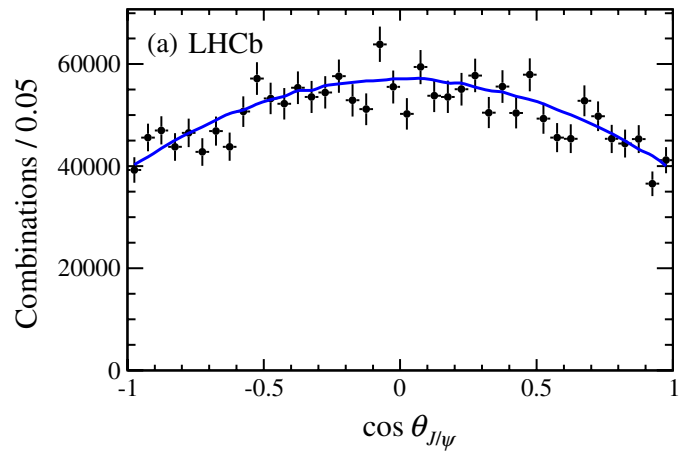

is defined within one full width of the $f_{2}^{\prime}(1525)$ mass. The background-subtracted efficiency-corrected distributions are shown in Figs. 20 and 21. The distributions are in good agreement with the fit model.

\section{E. Angular moments}

The angular moment distributions provide an additional way of visualizing the effects of different resonances and their interferences, similar to a partial wave analysis. This technique has been used in previous studies [8,34].

We define the angular moments $\left\langle Y_{l}^{0}\right\rangle$ as the efficiencycorrected and background-subtracted $K^{+} K^{-}$invariant mass distributions, weighted by orthogonal and normalized spherical harmonic functions $Y_{l}^{0}\left(\cos \theta_{K K}\right)$,

$\left\langle Y_{l}^{0}\right\rangle=\int_{-1}^{1} d \Gamma\left(m_{K K}, \cos \theta_{K K}\right) Y_{l}^{0}\left(\cos \theta_{K K}\right) d \cos \theta_{K K}$

If we assume that no $K^{+} K^{-}$partial-waves of a higher order than $D$-wave contribute, then we can express the differential decay rate, derived from Eq. (4) in terms of $S$-, $P$-, and $D$-waves including helicity 0 and \pm 1 components as

$$
\begin{aligned}
\frac{d \bar{\Gamma}}{d m_{K K} d \cos \theta_{K K}} \\
=2 \pi \mid \mathcal{S}_{0} Y_{0}^{0}\left(\cos \theta_{K K}\right)+\mathcal{P}_{0} e^{i \Phi_{P_{0}} Y_{1}^{0}\left(\cos \theta_{K K}\right)} \\
+\mathcal{D}_{0} e^{\left.i \Phi_{D_{0}} Y_{2}^{0}\left(\cos \theta_{K K}\right)\right|^{2}} \\
+2 \pi \mid \mathcal{P}_{ \pm 1} e^{i \Phi_{P_{ \pm 1}}} \sqrt{\frac{3}{8 \pi}} \sin \theta_{K K} \\
+\left.\mathcal{D}_{ \pm 1} e^{i \Phi_{D_{ \pm 1}}} \sqrt{\frac{15}{8 \pi}} \sin \theta_{K K} \cos \theta_{K K}\right|^{2},
\end{aligned}
$$

where $\mathcal{S}_{\lambda}, \mathcal{P}_{\lambda}, \mathcal{D}_{\lambda}$ and $\Phi_{k_{\lambda}}$ are real-valued functions of $m_{K K}$, and we have factored out the $S$-wave phase. We can then calculate the angular moments

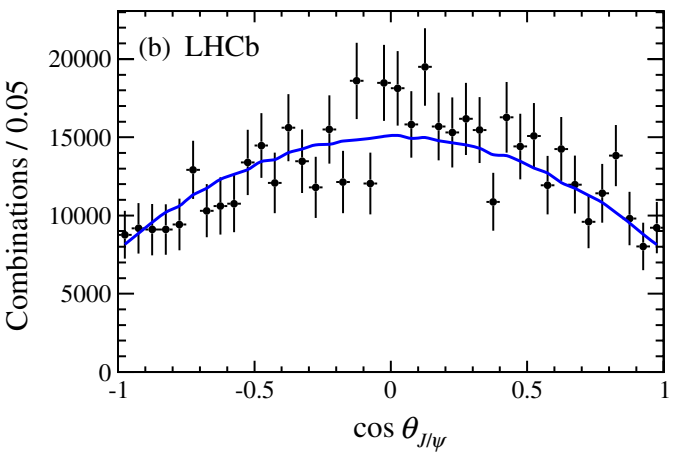

FIG. 20 (color online). Background-subtracted efficiency-corrected $\cos \theta_{J / \psi}$ helicity distributions: (a) in $\phi(1020)$ mass region $\left(\chi^{2} / \mathrm{ndf}=54.4 / 40\right)$, (b) in $f_{2}^{\prime}(1525)$ mass region $\left(\chi^{2} / \mathrm{ndf}=34.4 / 40\right)$. 

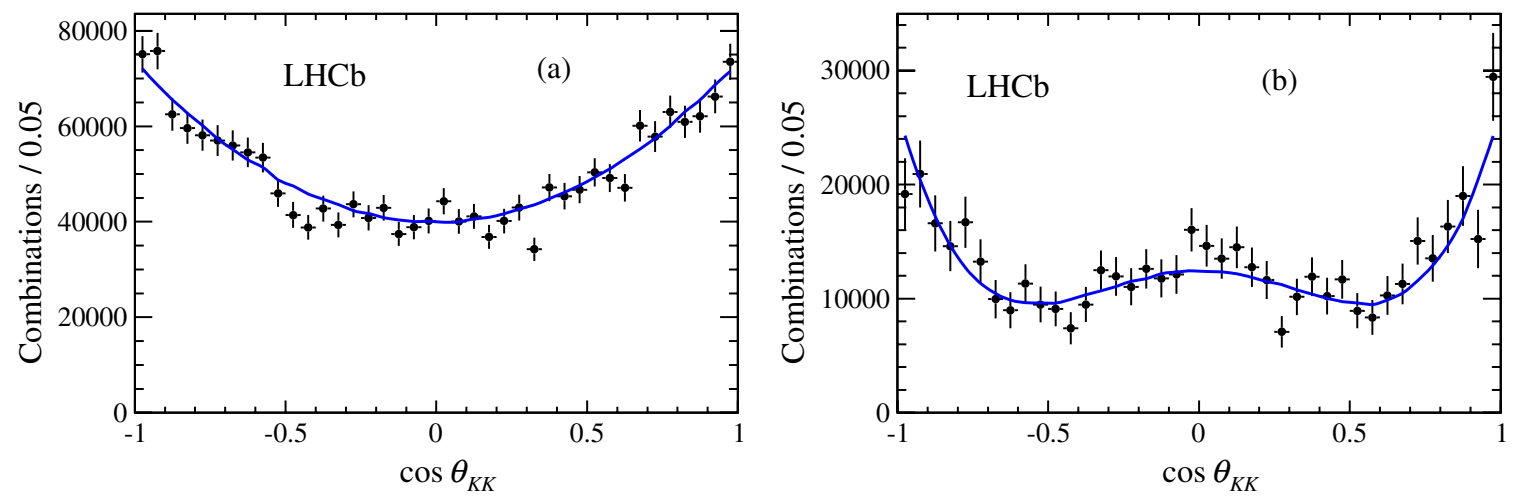

FIG. 21 (color online). Background-subtracted efficiency-corrected $\cos \theta_{K K}$ helicity distributions: (a) in $\phi(1020)$ mass region $\left(\chi^{2} /\right.$ ndf $\left.=57.4 / 40\right)$, (b) in $f_{2}^{\prime}(1525)$ mass region $\left(\chi^{2} /\right.$ ndf $\left.=43.4 / 40\right)$. The distributions are compatible with expectations for spin-1 and spin-2, respectively.
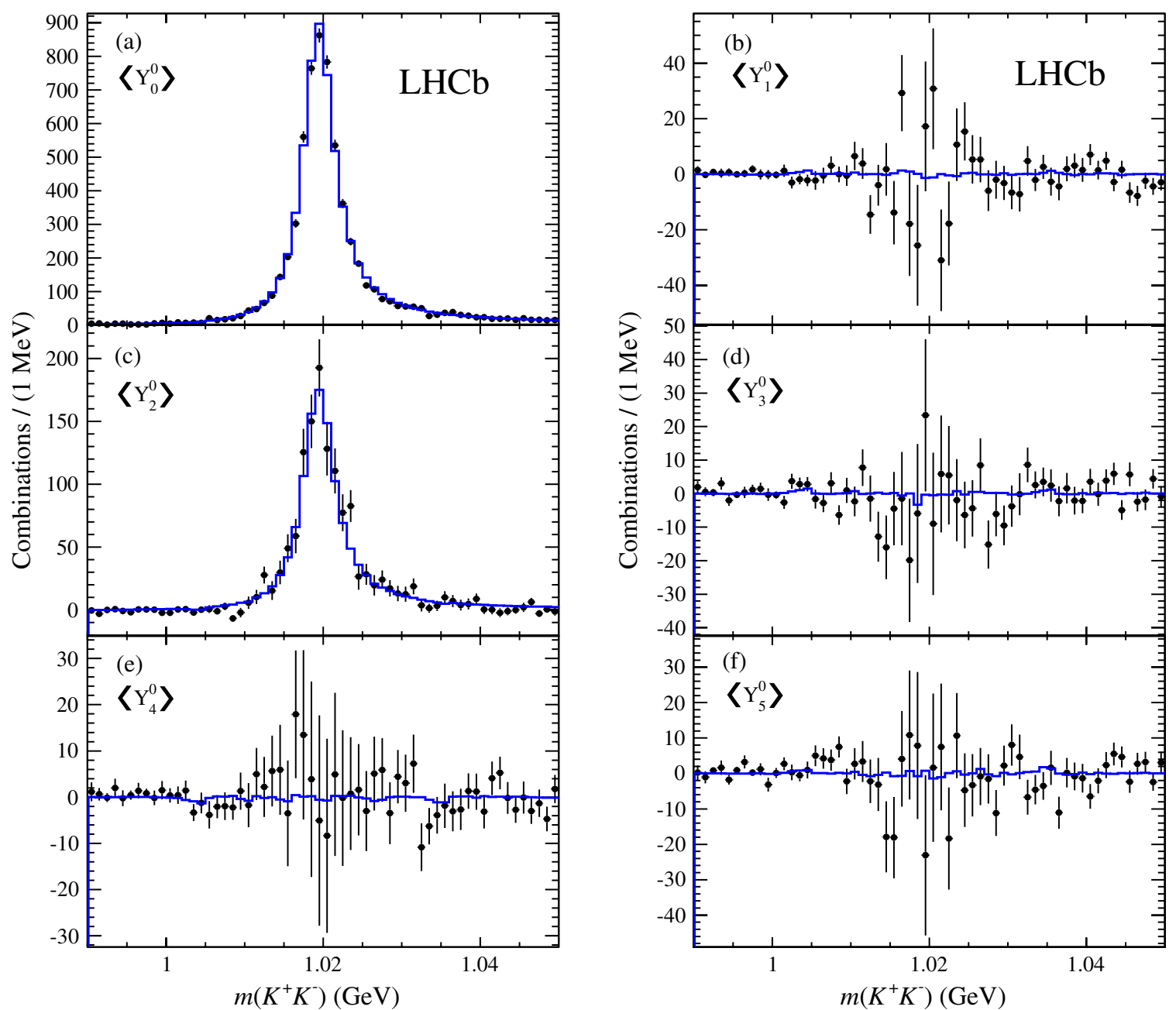

FIG. 22 (color online). Dependence of the spherical harmonic moments of $\cos \theta_{K K}$ as a function of the $K^{+} K^{-}$mass around the $\phi(1020)$ mass peak after efficiency corrections and background subtraction. The points with error bars are the data and the solid curves are derived from the fit model. 


$$
\begin{aligned}
& \sqrt{4 \pi}\left\langle Y_{0}^{0}\right\rangle=\mathcal{S}_{0}^{2}+\mathcal{P}_{0}^{2}+\mathcal{D}_{0}^{2}+\mathcal{P}_{ \pm 1}^{2}+\mathcal{D}_{ \pm 1}^{2} \\
& \sqrt{4 \pi}\left\langle Y_{1}^{0}\right\rangle=2 \mathcal{S}_{0} \mathcal{P}_{0} \cos \Phi_{P_{0}}+\frac{4}{\sqrt{5}} \mathcal{P}_{0} \mathcal{D}_{0} \cos \left(\Phi_{P_{0}}-\Phi_{D_{0}}\right)+8 \sqrt{\frac{3}{5}} \mathcal{P}_{ \pm 1} \mathcal{D}_{ \pm 1} \cos \left(\Phi_{P_{ \pm 1}}-\Phi_{D_{ \pm 1}}\right) \\
& \sqrt{4 \pi}\left\langle Y_{2}^{0}\right\rangle=\frac{2}{\sqrt{5}} \mathcal{P}_{0}^{2}+2 \mathcal{S}_{0} \mathcal{D}_{0} \cos \Phi_{D_{0}}+\frac{2 \sqrt{5}}{7} \mathcal{D}_{0}^{2}-\frac{1}{\sqrt{5}} \mathcal{P}_{ \pm 1}^{2}+\frac{\sqrt{5}}{7} \mathcal{D}_{ \pm 1}^{2} \\
& \sqrt{4 \pi}\left\langle Y_{3}^{0}\right\rangle=6 \sqrt{\frac{3}{35}} \mathcal{P}_{0} \mathcal{D}_{0} \cos \left(\Phi_{P_{0}}-\Phi_{D_{0}}\right)+\frac{6}{\sqrt{35}} \mathcal{P}_{ \pm 1} \mathcal{D}_{ \pm 1} \cos \left(\Phi_{P_{ \pm 1}}-\Phi_{D_{ \pm 1}}\right) \\
& \sqrt{4 \pi}\left\langle Y_{4}^{0}\right\rangle=\frac{6}{7} \mathcal{D}_{0}^{2}-\frac{4}{7} \mathcal{D}_{ \pm 1}^{2} .
\end{aligned}
$$

The angular moments for $l>4$ vanish. Figures 22 and 23 show the distributions of the angular moments for the fit model around $\pm 30 \mathrm{MeV}$ of the $\phi(1020)$ mass peak and above the $\phi(1020)$, respectively. In general the

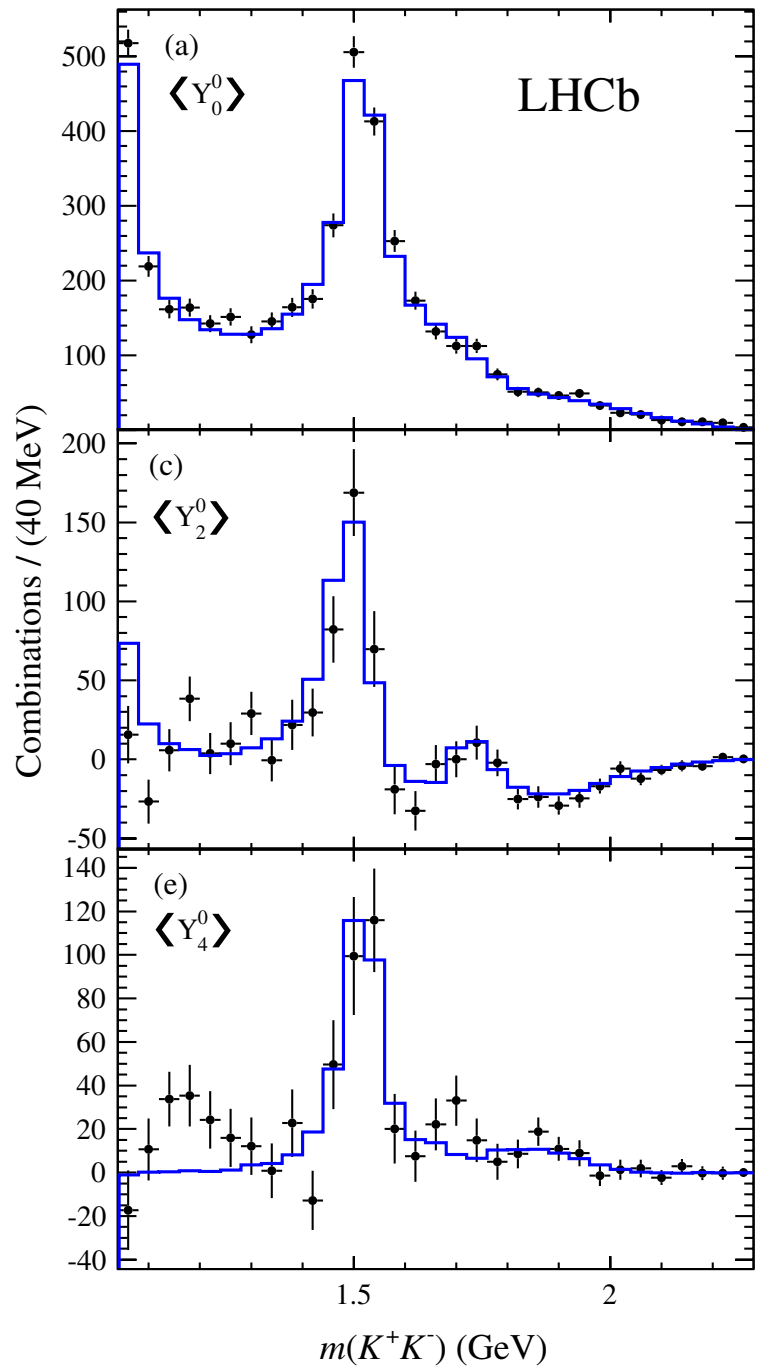

interpretation of these moments is that $\left\langle Y_{0}^{0}\right\rangle$ is the efficiency-corrected and background-subtracted event distribution, $\left\langle Y_{1}^{0}\right\rangle$ the sum of the interference between $S$-wave and $P$-wave, and $P$-wave and $D$-wave amplitudes, $\left\langle Y_{2}^{0}\right\rangle$ the

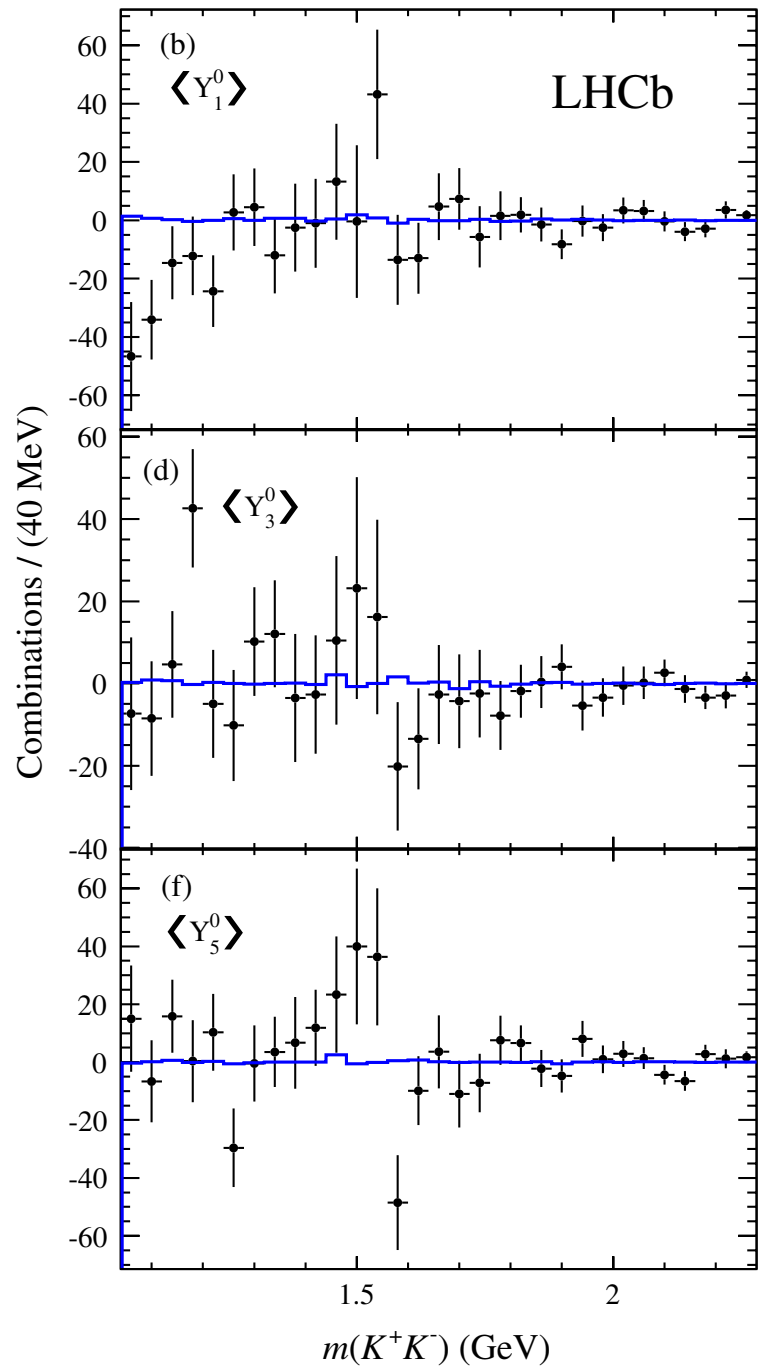

FIG. 23 (color online). Dependence of the spherical harmonic moments of $\cos \theta_{K K}$ as a function of the $K^{+} K^{-}$mass above $1050 \mathrm{MeV}$, after efficiency corrections and background subtraction. The points with error bars are the data and the solid curves are derived from the fit model. 
sum of $P$-wave, $D$-wave and the interference of $S$-wave and $D$-wave amplitudes, $\left\langle Y_{3}^{0}\right\rangle$ the interference between $P$-wave and $D$-wave amplitudes, and $\left\langle Y_{4}^{0}\right\rangle$ the $D$-wave. As discussed in Sec. VA, the average of $B_{s}^{0}$ and $\bar{B}_{s}^{0}$ cancels the interference terms that involve $\mathcal{P}_{0}$ and $\mathcal{D}_{ \pm}$. This causes the angular moments $\left\langle Y_{1}^{0}\right\rangle$ and $\left\langle Y_{3}^{0}\right\rangle$ to be zero when averaging over $B_{s}^{0}$ and $\bar{B}_{s}^{0}$ decays. We observe that the fit results well describe the moment distributions, except for the $\left\langle Y_{1}^{0}\right\rangle$ and $\left\langle Y_{4}^{0}\right\rangle$ values below $1.2 \mathrm{GeV}$. This may be the result of statistical fluctuations or imperfect modeling.

\section{F. Systematic uncertainties}

The sources of the systematic uncertainties on the results of the Dalitz plot analysis are summarized in
Table VII. The uncertainties due to the background parametrization are estimated by comparing the results from the best fit model with those when the background shape parameters are obtained from a fit to the lower sideband region only. The uncertainties in the efficiency are estimated by comparing the fit results when the efficiency parameters are changed by their statistical uncertainties and are added in quadrature. The effect on the fit fractions of changing the efficiency function is evaluated using a similar method to that used previously [8]. Briefly, we change the efficiency model by increasing the minimum IP $\chi^{2}$ requirement from 9 to 12.5 on both of the kaon candidates. This has the effect of increasing the $\chi^{2}$ of the fit to the angular distributions of $\bar{B}_{s}^{0} \rightarrow J / \psi \phi$ data by 1 unit. The new efficiency

TABLE VII. Absolute systematic uncertainties on the fit results.

\begin{tabular}{|c|c|c|c|c|}
\hline Item & Efficiency & Background & Fit model & Total \\
\hline$m_{f_{2}^{\prime}(1525)}(\mathrm{MeV})$ & 1.2 & 0.4 & $\begin{array}{l}+5.2 \\
-1.5\end{array}$ & $\begin{array}{l}+5.3 \\
-2.0\end{array}$ \\
\hline$\Gamma_{f_{2}^{\prime}(1525)}(\mathrm{MeV})$ & 4.7 & 0.5 & $\begin{array}{l}+8.6 \\
-1.8\end{array}$ & $\begin{array}{l}+9.8 \\
-5.0\end{array}$ \\
\hline \multicolumn{5}{|c|}{ Fit fractions $(\%)$} \\
\hline$\phi(1020) \lambda=0$ & 0.8 & 0 & $\begin{array}{l}+0.06 \\
-0.04\end{array}$ & \pm 0.8 \\
\hline$\phi(1020)|\lambda|=1$ & 1.3 & 0 & $\begin{array}{l}+0.4 \\
-0.1\end{array}$ & \pm 1.3 \\
\hline$f_{0}(980)$ & 1.7 & 0.4 & $\begin{array}{l}+2.1 \\
-1.7\end{array}$ & $\begin{array}{l}+2.8 \\
-2.5\end{array}$ \\
\hline$f_{0}(1370)$ & 0.3 & 0.02 & $\begin{array}{l}+0.2 \\
-1.2\end{array}$ & $\begin{array}{l}+0.3 \\
-1.3\end{array}$ \\
\hline$f_{2}^{\prime}(1525) \lambda=0$ & 1.5 & 0.2 & $\begin{array}{l}+1.8 \\
-0.5\end{array}$ & $\begin{array}{l}+2.4 \\
-1.6\end{array}$ \\
\hline$f_{2}^{\prime}(1525)|\lambda|=1$ & 1.1 & 0.4 & $\begin{array}{l}+1.3 \\
-0.8\end{array}$ & $\begin{array}{l}+1.8 \\
-1.4\end{array}$ \\
\hline$f_{2}(1640)|\lambda|=1$ & 0.1 & 0.1 & $\begin{array}{l}+0.7 \\
-0.9\end{array}$ & $\begin{array}{l}+0.7 \\
-0.9\end{array}$ \\
\hline$\phi(1680)|\lambda|=1$ & 0.3 & 0.1 & $\begin{array}{l}+4.4 \\
-0.1\end{array}$ & $\begin{array}{l}+4.4 \\
-0.3\end{array}$ \\
\hline$f_{2}(1750) \lambda=0$ & 0.3 & 0.1 & $\begin{array}{l}+1.0 \\
-0.5\end{array}$ & $\begin{array}{l}+1.0 \\
-0.6\end{array}$ \\
\hline$f_{2}(1750)|\lambda|=1$ & 1.6 & 0.3 & $\begin{array}{l}+1.5 \\
-2.9\end{array}$ & $\begin{array}{l}+2.2 \\
-3.3\end{array}$ \\
\hline$f_{2}(1950) \lambda=0$ & 0.1 & 0.04 & $\begin{array}{l}+0.2 \\
-0.4\end{array}$ & $\begin{array}{l}+0.2 \\
-0.4\end{array}$ \\
\hline$f_{2}(1950)|\lambda|=1$ & 2.1 & 0.6 & $\begin{array}{l}+1.1 \\
-3.1\end{array}$ & $\begin{array}{l}+2.5 \\
-3.8\end{array}$ \\
\hline Nonresonant & 1.7 & 0.4 & $\begin{array}{l}+0.9 \\
-1.5\end{array}$ & $\begin{array}{l}+2.0 \\
-2.2\end{array}$ \\
\hline$S$-wave within $\pm 12 \mathrm{MeV}$ of $\phi(1020)$ peak $(\%)$ & 0.02 & 0.02 & $\begin{array}{l}+0.2 \\
-0.1\end{array}$ & $\begin{array}{l}+0.2 \\
-0.1\end{array}$ \\
\hline \multicolumn{5}{|c|}{ Phases (degrees) } \\
\hline$f_{0}(980)$ & 19 & 8 & $\begin{array}{l}+14 \\
-8\end{array}$ & $\begin{array}{l}+25 \\
-22\end{array}$ \\
\hline$f_{0}(1370)$ & 6 & 1 & $\begin{array}{l}+6 \\
-5\end{array}$ & \pm 8 \\
\hline$f_{2}(1640)|\lambda|=1$ & 7 & 11 & $\begin{array}{l}+0 \\
-42\end{array}$ & $\begin{array}{l}+13 \\
-44\end{array}$ \\
\hline$\phi(1680)|\lambda|=1$ & 1 & 1 & $\begin{array}{l}+260 \\
-210\end{array}$ & $\begin{array}{l}+260 \\
-210\end{array}$ \\
\hline$f_{2}(1750) \lambda=0$ & 2 & 2 & $\begin{array}{l}+9 \\
-9\end{array}$ & \pm 9 \\
\hline$f_{2}(1750)|\lambda|=1$ & 12 & 10 & $\begin{array}{l}+2 \\
-70\end{array}$ & $\begin{array}{l}+16 \\
-70\end{array}$ \\
\hline$f_{2}(1950) \lambda=0$ & 13 & 1 & $\begin{array}{c}+108 \\
-14\end{array}$ & $\begin{array}{l}+110 \\
-20\end{array}$ \\
\hline$f_{2}(1950)|\lambda|=1$ & 77 & 26 & $\begin{array}{c}+130 \\
-1\end{array}$ & $\begin{array}{l}+150 \\
-80\end{array}$ \\
\hline Nonresonant & 18 & 7 & $\begin{array}{c}+12 \\
-5\end{array}$ & $\begin{array}{l}+23 \\
-20 \\
\end{array}$ \\
\hline
\end{tabular}


function is then applied to the data with the original minimum IP $\chi^{2}$ selection of 9, the likelihood is reevaluated and the uncertainties are estimated by comparing the results with the best fit model. The largest variations among these two efficiency categories are included in the uncertainty.

We estimate additional uncertainties by comparing the results when one more resonance is added to the best fit model. The uncertainties due to the line shape of the contributing resonances with fixed mass and width parameters are estimated by varying them individually in the fit according to their combined statistical and systematic uncertainties added in quadrature. We compare the results with the best fit and add them in quadrature to estimate the uncertainties due to the line shape.

Another source of systematic uncertainty is the value we choose for $L_{B}$, the orbital angular momentum in the $\bar{B}_{s}^{0}$ decay. If $L_{R}$ equals zero then $L_{B}$ equals zero. If, however, $L_{R}$ is 1 then $L_{B}$ can either be 0 or 1 , and if $L_{R}$ is $2, L_{B}$ can be 1,2 or 3 . For our best fit we do not allow multiple values for $L_{B}$ but choose the lowest allowed value. To estimate the systematic uncertainties due to the choice of $L_{B}$, we repeat the fit changing the default value of $L_{B}$, in turn, to each higher allowed value and compare the fit results with the best fit. The differences are grouped into the fit model category, and we assign the largest variations as the systematic uncertainties. These later two categories often give in asymmetric uncertainties.

\section{ABSOLUTE BRANCHING FRACTIONS}

Branching fractions are measured from ratios of the decay rates of interest normalized to the well-established decay mode $B^{-} \rightarrow J / \psi K^{-}$. This decay mode, in addition to having a well-measured branching fraction, has the advantage of having two muons in the final state and hence the same triggers as the $\bar{B}_{s}^{0}$ decay. However, we require knowledge of the $\bar{B}_{s}^{0} / B^{-}$production ratio. For this we assume isospin invariance and use the $\bar{B}_{s}^{0} / \bar{B}^{0}$ production ratio $f_{s} / f_{d}=0.256 \pm 0.020$, given in Ref. [35]. The branching fractions are calculated using

$$
\mathcal{B}\left(\bar{B}_{s}^{0} \rightarrow J / \psi X\right)=\frac{N_{\bar{B}_{s}^{0}} / \epsilon_{\bar{B}_{s}^{0}}}{N_{B^{-}} / \epsilon_{B^{-}}} \times \mathcal{B}\left(B^{-} \rightarrow J / \psi K^{-}\right) \times \frac{1}{f_{s} / f_{d}},
$$

where $X$ indicates a specific $K^{+} K^{-}$state, $N$ represents the yield of the decay of interest, and $\epsilon$ corresponds to the overall efficiency. We form an average of $\mathcal{B}\left(B^{-} \rightarrow J / \psi K^{-}\right)=(10.18 \pm 0.42) \times 10^{-4}$ using the recent Belle [36] and BABAR [37] measurements, corrected to take into account different rates of $B^{+} B^{-}$and $B^{0} \bar{B}^{0}$ pair production from $\Upsilon(4 S)$ using $\frac{\Gamma\left(B^{+} B^{-}\right)}{\Gamma\left(B^{0} \bar{B}^{0}\right)}=1.055 \pm 0.025[9]$.

The detection efficiency is obtained from simulation and is a product of the geometrical acceptance of the detector, the combined reconstruction and selection efficiency and the trigger efficiency. The efficiency also includes the efficiency of the Dalitz plot model for the case of $\bar{B}_{s}^{0} \rightarrow J / \psi K^{+} K^{-}$, where the best fit model is used. The detection efficiencies and their various correction factors are given in Table VIII. To ensure that the $p$ and $p_{\mathrm{T}}$ distributions of the generated $B$ meson are correct we weight the $\bar{B}_{s}^{0}$ simulations using $\bar{B}_{s}^{0} \rightarrow J / \psi \phi(1020)$ data and the $B^{-}$simulations using $B^{-} \rightarrow J / \psi K^{-}$data. Since the control channel has a different number of charged tracks than the decay channel, we weight the simulations with the tracking efficiency ratio by comparing the data and simulations in bins of the track's $p$ and $p_{\mathrm{T}}$. We further weight the $\bar{B}_{s}^{0} \rightarrow J / \psi K^{+} K^{-}$simulation, using the PDG value of $\bar{B}_{s}^{0}$ lifetime, $(1.497 \pm 0.015) \times 10^{-12} \mathrm{~s}[9]$, as input.

The resulting branching fractions are

$$
\begin{aligned}
\mathcal{B}\left(\bar{B}_{s}^{0}\right. & \left.\rightarrow J / \psi K^{+} K^{-}\right) \\
& =(7.70 \pm 0.08 \pm 0.39 \pm 0.60) \times 10^{-4}, \\
\mathcal{B}\left(\bar{B}_{s}^{0}\right. & \rightarrow J / \psi \phi(1020)) \\
& =(10.50 \pm 0.13 \pm 0.64 \pm 0.82) \times 10^{-4}, \\
\mathcal{B}\left(\bar{B}_{s}^{0}\right. & \left.\rightarrow J / \psi f_{2}^{\prime}(1525)\right) \\
& =\left(2.61 \pm 0.20_{-0.46}^{+0.52} \pm 0.20\right) \times 10^{-4},
\end{aligned}
$$

TABLE VIII. Detector efficiencies determined from simulation and the correction factors.

\begin{tabular}{lcc}
\hline \hline Item & $J / \psi K^{+} K^{-}$ & $J / \psi K^{-}$ \\
\hline Detection efficiency (\%) & $1.061 \pm 0.004$ & $2.978 \pm 0.011$ \\
& $\quad$ Correction factors & \\
Tracking efficiency & $0.999 \pm 0.010$ & $1.003 \pm 0.010$ \\
PID & $0.819 \pm 0.008$ & $0.974 \pm 0.005$ \\
$p$ and $p_{\mathrm{T}}$ & $1.077 \pm 0.005$ & $1.053 \pm 0.005$ \\
$\bar{B}_{s}^{0}$ lifetime & $0.993 \pm 0.015$ & $\ldots$ \\
Total efficiency $(\%)$ & $0.887 \pm 0.004 \pm 0.018$ & $3.065 \pm 0.012 \pm 0.038$ \\
\hline \hline
\end{tabular}


TABLE IX. Relative systematic uncertainties on branching fractions (\%).

\begin{tabular}{lccc}
\hline \hline Item & $J / \psi K^{+} K^{-}$ & $J / \psi \phi(1020)$ & $J / \psi f_{2}^{\prime}(1525)$ \\
\hline Tracking efficiency & 1.0 & 1.0 & 1.0 \\
Material and physical effects & 2.0 & 2.0 & 2.0 \\
PID efficiency & 1.0 & 1.0 & 1.0 \\
$\bar{B}_{s}^{0} p$ and $p_{\mathrm{T}}$ distributions & 0.5 & 0.5 & 0.5 \\
$B^{-} p$ and $p_{\mathrm{T}}$ distributions & 0.5 & 0.5 & 0.5 \\
$\bar{B}_{s}^{0}$ lifetime & 1.5 & 1.5 & 1.5 \\
Efficiency function & 0.02 & 0.02 & 0.02 \\
$\mathcal{B}\left(\phi(1020) \rightarrow K^{+} K^{-}\right)$ & $\ldots$ & 1.0 & $\ldots$ \\
$\mathcal{B}\left(f_{2}^{\prime}(1525) \rightarrow K^{+} K^{-}\right)$ & $\ldots$ & $\ldots$ & 2.5 \\
$\mathcal{B}\left(B^{-} \rightarrow J / \psi K^{-}\right)$ & 4.1 & 4.1 & 4.1 \\
& Contributions from Dalitz analysis & \\
Efficiency & $\ldots$ & 3.1 & 12.3 \\
Background & $\ldots$ & 0 & 1.3 \\
Fit model & $\ldots$ & ${ }^{+0.7}$ & -14.6 \\
Sum in quadrature of items above & 5.0 & -6.1 & +17.7 \\
$f_{s} / f_{d}$ & 7.8 & 7.8 & 7.8 \\
\hline \hline
\end{tabular}

where the branching fractions $\mathcal{B}\left(\phi(1020) \rightarrow K^{+} K^{-}\right)=$ $(48.9 \pm 0.5) \%$ and $\mathcal{B}\left(f_{2}^{\prime}(1525) \rightarrow K^{+} K^{-}\right)=(44.4 \pm 1.1) \%$ are used [9]. Here the first uncertainty in each case is statistical, the second is systematic and the third reflects the uncertainty due to $f_{s} / f_{d}$. Note that these are the time-integrated branching fractions. Results on the polarization fractions of $\bar{B}_{s}^{0} \rightarrow J / \psi \phi(1020)$ from a time-dependent analysis will be forthcoming in a separate publication [38]. The ratio of $\mathcal{B}\left(\bar{B}_{s}^{0} \rightarrow J / \psi f_{2}^{\prime}(1525)\right) /$ $\mathcal{B}\left(\bar{B}_{s}^{0} \rightarrow J / \psi \phi(1020)\right)$ is consistent with our previous result [10], D0 [11], and the Belle result [39]. The current PDG value of $\mathcal{B}\left(\bar{B}_{s}^{0} \rightarrow J / \psi \phi(1020)\right)=(1.4 \pm 0.5) \times 10^{-3}$ is dominated by the CDF measurement [40]. Our measured value is in good agreement with this measurement and also the most recent yet unpublished values measured by CDF [41] and Belle [39]. The Belle collaboration has also recently reported the branching fraction of $\mathcal{B}\left(\bar{B}_{s}^{0} \rightarrow J / \psi K^{+} K^{-}\right)$[39], where $\bar{B}_{s}^{0} \rightarrow J / \psi \phi(1020)$ is excluded.

The systematic uncertainty on the branching fraction has several contributions listed in Table IX. Since the branching fractions are measured with respect to $B^{-} \rightarrow J / \psi K^{-}$ which has a different number of charged tracks than the decays of interest, a $1 \%$ systematic uncertainty is assigned due to differences in the tracking performance between data and simulation. Another $2 \%$ uncertainty is assigned for the additional kaon which is due to decay in flight, large multiple scatterings and hadronic interactions along the track. Using the PDG value for the $\bar{B}_{s}^{0}$ lifetime [9] as input gives rise to an additional $1.5 \%$ systematic uncertainty. Small uncertainties are introduced if the simulation does not have the correct $B$ meson kinematic distributions. We are relatively insensitive to any of these differences in the $B$ meson $p$ and $p_{\mathrm{T}}$ distributions since we are measuring the relative rates. By varying the $p$ and $p_{\mathrm{T}}$ distributions we see at most a change of $0.5 \%$. There is a $1 \%$ systematic uncertainty assigned for the relative particle identification efficiencies. An uncertainty of $0.02 \%$ is included due to the change of the efficiency function Eq. (20). Three additional uncertainties are considered in the branching fractions of $\mathcal{B}\left(\bar{B}_{s}^{0} \rightarrow J / \psi \phi(1020)\right)$ and $\mathcal{B}\left(\bar{B}_{s}^{0} \rightarrow J / \psi f_{2}^{\prime}(1525)\right)$ as these are measured from the fit fractions of the Dalitz plot analysis. The total systematic uncertainty is obtained by adding each source of systematic uncertainty in quadrature as they are uncorrelated.

\section{CONCLUSIONS}

We have determined the final state composition of the $\bar{B}_{s}^{0} \rightarrow J / \psi K^{+} K^{-}$decay channel using a modified Dalitz plot analysis where we include the decay angle of the $J / \psi$. The largest contribution is the $\phi(1020)$ resonance, along with other $S$-, $P$ - and $D$-wave $K^{+} K^{-}$states, and a nonresonant $K^{+} K^{-}$contribution. All of the components are listed in Table IV. The mass and width of the $f_{2}^{\prime}(1525)$ resonance are measured as

$$
\begin{aligned}
& m_{f_{2}^{\prime}(1525)}=1522.2 \pm 2.8_{-2.0}^{+5.3} \mathrm{MeV}, \\
& \Gamma_{f_{2}^{\prime}(1525)}=84 \pm 6_{-5}^{+10} \mathrm{MeV} .
\end{aligned}
$$

We also observe a significant $S$-wave component that is present over the entire $K^{+} K^{-}$mass region. Within $\pm 12 \mathrm{MeV}$ of the $\phi(1020)$ mass it is $\left(1.1 \pm 0.1_{-0.1}^{+0.2}\right) \%$ of the yield, and can affect precision $C P$ violation measurements [12]. Finally we determine the absolute branching fractions 


$$
\begin{aligned}
\mathcal{B}\left(\bar{B}_{s}^{0}\right. & \left.\rightarrow J / \psi K^{+} K^{-}\right) \\
& =(7.70 \pm 0.08 \pm 0.39 \pm 0.60) \times 10^{-4}, \\
\mathcal{B}\left(\bar{B}_{s}^{0}\right. & \rightarrow J / \psi \phi(1020)) \\
& =(10.50 \pm 0.13 \pm 0.64 \pm 0.82) \times 10^{-4}, \\
\mathcal{B}\left(\bar{B}_{s}^{0}\right. & \left.\rightarrow J / \psi f_{2}^{\prime}(1525)\right) \\
& =\left(2.61 \pm 0.20_{-0.46}^{+0.52} \pm 0.20\right) \times 10^{-4},
\end{aligned}
$$

where the first uncertainty in each case is statistical, the second is systematic and the third due to $f_{s} / f_{d}$. These results provide a good understanding of the $J / \psi K^{+} K^{-}$final state in $\bar{B}_{s}^{0}$ decays over the entire kinematically allowed region. The $J / \psi f_{2}^{\prime}(1525)$ results supersede those of Ref. [10]. This decay mode offers the opportunity for additional measurements of $C P$ violation [42].

\section{ACKNOWLEDGMENTS}

We express our gratitude to our colleagues in the CERN accelerator departments for the excellent performance of the LHC. We thank the technical and administrative staff at the $\mathrm{LHCb}$ institutes. We acknowledge support from CERN and from the national agencies: CAPES, CNPq, FAPERJ and FINEP (Brazil); NSFC (China); CNRS/ IN2P3 and Region Auvergne (France); BMBF, DFG, HGF and MPG (Germany); SFI (Ireland); INFN (Italy); FOM and NWO (Netherlands); SCSR (Poland); ANCS/ IFA (Romania); MinES, Rosatom, RFBR and NRC "Kurchatov Institute" (Russia); MinECo, XuntaGal and GENCAT (Spain); SNSF and SER (Switzerland); NAS Ukraine (Ukraine); STFC (United Kingdom); NSF (USA). We also acknowledge the support received from the ERC under FP7. The Tier1 computing centers are supported by IN2P3 (France), KIT and BMBF (Germany), INFN (Italy), NWO and SURF (Netherlands), PIC (Spain), GridPP (United Kingdom). We are thankful for the computing resources put at our disposal by Yandex LLC (Russia), as well as to the communities behind the multiple open source software packages that we depend on.
[1] R. Aaij et al. (LHCb Collaboration), Phys. Lett. B 713, 378 (2012).

[2] R. Aaij et al. (LHCb Collaboration), Phys. Rev. Lett. 108, 101803 (2012).

[3] R. Aaij et al. (LHCb Collaboration), Report No. LHCbCONF-2012-002.

[4] T. Aaltonen et al. (CDF Collaboration), Phys. Rev. D 85, 072002 (2012).

[5] V. M. Abazov et al. (D0 Collaboration), Phys. Rev. D 85, 032006 (2012).

[6] T. Aaltonen et al. (CDF Collaboration), Phys. Rev. Lett. 109, 171802 (2012).

[7] G. Aad et al. (ATLAS Collaboration), J. High Energy Phys. 12 (2012) 072.

[8] R. Aaij et al. (LHCb Collaboration), Phys. Rev. D 86, 052006 (2012).

[9] J. Beringer et al. (Particle Data Group), Phys. Rev. D 86, 010001 (2012).

[10] R. Aaij et al. (LHCb Collaboration), Phys. Rev. Lett. 108, 151801 (2012).

[11] V. M. Abazov et al. (D0 collaboration), Phys. Rev. D 86, 092011 (2012).

[12] S. Stone and L. Zhang, Phys. Rev. D 79, 074024 (2009).

[13] R. H. Dalitz, Philos. Mag. 44, 1068 (1953).

[14] A. Alves, Jr. et al. (LHCb Collaboration), J. Instrum. 3, S08005 (2008).

[15] R. Aaij et al., arXiv:1211.3055.

[16] T. Sjöstrand, S. Mrenna, and P. Skands, J. High Energy Phys. 05 (2006) 026.

[17] I. Belyaev et al., in Handling of the Generation of Primary Events in GAUSS, the LHCb Simulation Framework,
Nuclear Science Symposium Conference Record No. (NSS/MIC) (IEEE, New York, 2010), p. 1155.

[18] S. Agostinelli et al. (GEANT4 Collaboration), Nucl. Instrum. Methods Phys. Res., Sect. A 506, 250 (2003); J. Allison et al. (GEANT4 Collaboration), Nucl. Sci. Res. Conf. Ser. 53, 270 (2006).

[19] M. Clemencic, G. Corti, S. Easo, C. R. Jones, S. Miglioranzi, M. Pappagallo, and P. Robbe, J. Phys. Conf. Ser. 331, 032023 (2011).

[20] D. Lange, Nucl. Instrum. Methods Phys. Res., Sect. A 462, 152 (2001).

[21] R. Aaij et al. (LHCb Collaboration), Phys. Lett. B 698, 115 (2011).

[22] G. N. Fleming, Phys. Rev. 135, B551 (1964).

[23] D. Morgan, Phys. Rev. 166, 1731 (1968).

[24] R. Mizuk et al. (Belle Collaboration), Phys. Rev. D 78, 072004 (2008).

[25] J.M. Blatt and V.F. Weisskopf, Theoretical Nuclear Physics (Wiley/Springer-Verlag, New York, 1952).

[26] S. Kopp et al. (CLEO Collaboration), Phys. Rev. D 63, 092001 (2001).

[27] S. M. Flatté, Phys. Lett. 63B, 228 (1976).

[28] K. Abe et al. (Belle Collaboration), Eur. Phys. J. C 32, 323 (2003).

[29] M. Acciarri et al. (L3 Collaboration), Phys. Lett. B 501, 173 (2001).

[30] S. Baker and R. D. Cousins, Nucl. Instrum. Methods Phys. Res. 221, 437 (1984).

[31] M. Ablikim et al. (BES Collaboration), Phys. Lett. B 607, 243 (2005)

[32] Antimo Palano and Marco Papagallo (private communication). 
[33] P. del Amo Sanchez et al. (BABAR Collaboration), Phys. Rev. D 83, 052001 (2011).

[34] J. P. Lees et al. (BABAR Collaboration), Phys. Rev. D 85, 112010 (2012).

[35] R. Aaij et al. (LHCb Collaboration), Report No. LHCbPAPER-2012-037; Phys. Rev. D 85, 032008 (2012).

[36] K. Abe et al. (Belle Collaboration), Phys. Rev. D 67, 032003 (2003).

[37] B. Aubert et al. (BABAR Collaboration), Phys. Rev. Lett. 94, 141801 (2005).
[38] R. Aaij et al. (LHCb Collaboration), Report No. LHCbPAPER-2013-001.

[39] F. Thorne (Belle Collaboration), in $B_{s}$ Measurements at Belle (ICHEP, Melbourne, 2012).

[40] F. Abe et al. (CDF Collaboration), Phys. Rev. D 54, 6596 (1996).

[41] CDF Collaboration, Measurement of $\mathcal{B}\left(B_{s}^{0} \rightarrow J / \psi \phi\right)$ and extraction of the fragmentation functions, Public Note 10795.

[42] C. Sharma and R. Sinha, Phys. Rev. D 73, 014016 (2006); L. Zhang and S. Stone, Phys. Lett. B 719, 383 (2013).

R. Aaij, ${ }^{38}$ C. Abellan Beteta, ${ }^{33, a}$ A. Adametz,${ }^{11}$ B. Adeva, ${ }^{34}$ M. Adinolfi, ${ }^{43}$ C. Adrover, ${ }^{6}$ A. Affolder, ${ }^{49}$ Z. Ajaltouni, ${ }^{5}$ J. Albrecht, ${ }^{35}$ F. Alessio, ${ }^{35}$ M. Alexander, ${ }^{48}$ S. Ali, ${ }^{38}$ G. Alkhazov, ${ }^{27}$ P. Alvarez Cartelle, ${ }^{34}$ A. A. Alves, Jr., ${ }^{22,35}$ S. Amato, ${ }^{2}$ Y. Amhis, ${ }^{7}$ L. Anderlini, ${ }^{17, b}$ J. Anderson, ${ }^{37}$ R. Andreassen,${ }^{57}$ R. B. Appleby, ${ }^{51}$ O. Aquines Gutierrez, ${ }^{10}$ F. Archilli, ${ }^{18}$ A. Artamonov, ${ }^{32}$ M. Artuso, ${ }^{53}$ E. Aslanides, ${ }^{6}$ G. Auriemma, ${ }^{22, c}$ S. Bachmann, ${ }^{11}$ J. J. Back, ${ }^{45}$ C. Baesso, ${ }^{54}$ V. Balagura, ${ }^{28}$ W. Baldini, ${ }^{16}$ R. J. Barlow, ${ }^{51}$ C. Barschel, ${ }^{35}$ S. Barsuk, ${ }^{7}$ W. Barter, ${ }^{44}$ A. Bates, ${ }^{48}$ Th. Bauer, ${ }^{38}$ A. Bay, ${ }^{36}$ J. Beddow, ${ }^{48}$ I. Bediaga, ${ }^{1}$ S. Belogurov, ${ }^{28}$ K. Belous, ${ }^{32}$ I. Belyaev ${ }^{28}$ E. Ben-Haim, ${ }^{8}$ M. Benayoun, ${ }^{8}$ G. Bencivenni, ${ }^{18}$ S. Benson, ${ }^{47}$ J. Benton, ${ }^{43}$ A. Berezhnoy, ${ }^{29}$ R. Bernet, ${ }^{37}$ M.-O. Bettler,${ }^{44}$ M. van Beuzekom, ${ }^{38}$ A. Bien, ${ }^{11}$ S. Bifani, ${ }^{12}$ T. Bird, ${ }^{51}$ A. Bizzeti, ${ }^{17, d}$ P. M. Bjørnstad, ${ }^{51}$ T. Blake, ${ }^{35}$ F. Blanc, ${ }^{36}$ C. Blanks, ${ }^{50}$ J. Blouw, ${ }^{11}$ S. Blusk, ${ }^{53}$ A. Bobrov,${ }^{31}$ V. Bocci, ${ }^{22}$ A. Bondar,${ }^{31}$ N. Bondar, ${ }^{27}$ W. Bonivento, ${ }^{15}$ S. Borghi, ${ }^{51}$ A. Borgia, ${ }^{53}$ T. J. V. Bowcock, ${ }^{49}$ E. Bowen, ${ }^{37}$ C. Bozzi, ${ }^{16}$ T. Brambach,,${ }^{9}$ J. van den Brand,${ }^{39}$ J. Bressieux,${ }^{36}$ D. Brett, ${ }^{51}$ M. Britsch, ${ }^{10}$ T. Britton, ${ }^{53}$ N. H. Brook,${ }^{43}$ H. Brown, ${ }^{49}$ A. Büchler-Germann, ${ }^{37}$ I. Burducea,${ }^{26}$ A. Bursche, ${ }^{37}$ J. Buytaert, ${ }^{35}$ S. Cadeddu, ${ }^{15}$ O. Callot, ${ }^{7}$ M. Calvi, ${ }^{20, e}$ M. Calvo Gomez, ${ }^{33, a}$ A. Camboni,${ }^{33}$ P. Campana, ${ }^{18,35}$ A. Carbone, ${ }^{14, \mathrm{f}}$ G. Carboni, ${ }^{21, \mathrm{~g}}$ R. Cardinale, ${ }^{19, \mathrm{~h}}$ A. Cardini, ${ }^{15}$ H. Carranza-Mejia, ${ }^{47}$ L. Carson, ${ }^{50}$ K. Carvalho Akiba, ${ }^{2}$ G. Casse, ${ }^{49}$ M. Cattaneo, ${ }^{35}$ Ch. Cauet, ${ }^{9}$ M. Charles, ${ }^{52}$ Ph. Charpentier, ${ }^{35}$ P. Chen, ${ }^{3,36}$ N. Chiapolini,${ }^{37}$ M. Chrzaszcz, ${ }^{23}$ K. Ciba, ${ }^{35}$ X. Cid Vidal,${ }^{34}$ G. Ciezarek,${ }^{50}$ P. E. L. Clarke, ${ }^{47}$ M. Clemencic,${ }^{35}$ H. V. Cliff, ${ }^{44}$ J. Closier,${ }^{35}$ C. Coca, ${ }^{26}$ V. Coco, ${ }^{38}$ J. Cogan,${ }^{6}$ E. Cogneras, ${ }^{5}$ P. Collins, ${ }^{35}$ A. Comerma-Montells,${ }^{33}$ A. Contu, ${ }^{15}$ A. Cook,${ }^{43}$ M. Coombes, ${ }^{43}$ G. Corti,${ }^{35}$ B. Couturier, ${ }^{35}$ G. A. Cowan, ${ }^{36}$ D. Craik,${ }^{45}$ S. Cunliffe,${ }^{50}$ R. Currie, ${ }^{47}$ C. D' Ambrosio, ${ }^{35}$ P. David, ${ }^{8}$ P. N. Y. David, ${ }^{38}$ I. De Bonis, ${ }^{4}$ K. De Bruyn, ${ }^{38}$ S. De Capua, ${ }^{51}$ M. De Cian,${ }^{37}$ J. M. De Miranda, ${ }^{1}$ L. De Paula, ${ }^{2}$ W. De Silva, ${ }^{57}$ P. De Simone, ${ }^{18}$ D. Decamp, ${ }^{4}$ M. Deckenhoff, ${ }^{9}$ H. Degaudenzi, ${ }^{36,35}$ L. Del Buono, ${ }^{8}$ C. Deplano, ${ }^{15}$ D. Derkach,${ }^{14}$ O. Deschamps,${ }^{5}$ F. Dettori, ${ }^{39}$ A. Di Canto, ${ }^{11}$ J. Dickens, ${ }^{44}$ H. Dijkstra, ${ }^{35}$ P. Diniz Batista, ${ }^{1}$ M. Dogaru, ${ }^{26}$ F. Domingo Bonal, ${ }^{33, a}$ S. Donleavy, ${ }^{49}$ F. Dordei,${ }^{11}$ A. Dosil Suárez, ${ }^{34}$ D. Dossett, ${ }^{45}$ A. Dovbnya ${ }^{40}$ F. Dupertuis, ${ }^{36}$ R. Dzhelyadin,${ }^{32}$ A. Dziurda, ${ }^{23}$ A. Dzyuba, ${ }^{27}$ S. Easo, ${ }^{46,35}$ U. Egede,${ }^{50}$ V. Egorychev, ${ }^{28}$ S. Eidelman, ${ }^{31}$ D. van Eijk,${ }^{38}$ S. Eisenhardt,${ }^{47}$ U. Eitschberger, ${ }^{9}$ R. Ekelhof, ${ }^{9}$ L. Eklund, ${ }^{48}$ I. El Rifai, ${ }^{5}$ Ch. Elsasser, ${ }^{37}$

D. Elsby ${ }^{42}$ A. Falabella, ${ }^{14, \mathrm{i}}$ C. Färber, ${ }^{11}$ G. Fardell,${ }^{47}$ C. Farinelli, ${ }^{38}$ S. Farry,${ }^{12}$ V. Fave,${ }^{36}$ D. Ferguson, ${ }^{47}$

V. Fernandez Albor, ${ }^{34}$ F. Ferreira Rodrigues, ${ }^{1}$ M. Ferro-Luzzi, ${ }^{35}$ S. Filippov, ${ }^{30}$ C. Fitzpatrick,${ }^{35}$ M. Fontana,${ }^{10}$

F. Fontanelli, ${ }^{19, \mathrm{~h}}$ R. Forty, ${ }^{35}$ O. Francisco, ${ }^{2}$ M. Frank,${ }^{35}$ C. Frei, ${ }^{35}$ M. Frosini, ${ }^{17, b}$ S. Furcas, ${ }^{20}$ E. Furfaro, ${ }^{21}$ A. Gallas Torreira, ${ }^{34}$ D. Galli, ${ }^{14, f}$ M. Gandelman, ${ }^{2}$ P. Gandini, ${ }^{52}$ Y. Gao, ${ }^{3}$ J. Garofoli, ${ }^{53}$ P. Garosi, ${ }^{51}$ J. Garra Tico, ${ }^{44}$ L. Garrido, ${ }^{33}$ C. Gaspar, ${ }^{35}$ R. Gauld,${ }^{52}$ E. Gersabeck, ${ }^{11}$ M. Gersabeck, ${ }^{51}$ T. Gershon, ${ }^{45,35}$ Ph. Ghez, ${ }^{4}$ V. Gibson, ${ }^{44}$ V. V. Gligorov, ${ }^{35}$ C. Göbel,${ }^{54}$ D. Golubkov, ${ }^{28}$ A. Golutvin, ${ }^{50,28,35}$ A. Gomes, ${ }^{2}$ H. Gordon, ${ }^{52}$ M. Grabalosa Gándara, ${ }^{5}$ R. Graciani Diaz, ${ }^{33}$ L. A. Granado Cardoso, ${ }^{35}$ E. Graugés, ${ }^{33}$ G. Graziani, ${ }^{17}$ A. Grecu ${ }^{26}$ E. Greening, ${ }^{52}$ S. Gregson, ${ }^{44}$ O. Grünberg, ${ }^{55}$ B. Gui,${ }^{53}$ E. Gushchin,${ }^{30}$ Yu. Guz,${ }^{32}$ T. Gys,${ }^{35}$ C. Hadjivasiliou, ${ }^{53}$ G. Haefeli, ${ }^{36}$ C. Haen, ${ }^{35}$ S. C. Haines, ${ }^{44}$ S. Hall, ${ }^{50}$ T. Hampson, ${ }^{43}$ S. Hansmann-Menzemer, ${ }^{11}$ N. Harnew, ${ }^{52}$ S. T. Harnew, ${ }^{43}$ J. Harrison, ${ }^{51}$ P. F. Harrison, ${ }^{45}$ T. Hartmann,${ }^{55}$ J. He ${ }^{7}$ V. Heijne,${ }^{38}$ K. Hennessy, ${ }^{49}$ P. Henrard,${ }^{5}$ J. A. Hernando Morata, ${ }^{34}$

E. van Herwijnen, ${ }^{35}$ E. Hicks, ${ }^{49}$ D. Hill, ${ }^{52}$ M. Hoballah, ${ }^{5}$ C. Hombach, ${ }^{51}$ P. Hopchev, ${ }^{4}$ W. Hulsbergen, ${ }^{38}$ P. Hunt, ${ }^{52}$ T. Huse,${ }^{49}$ N. Hussain, ${ }^{52}$ D. Hutchcroft, ${ }^{49}$ D. Hynds, ${ }^{48}$ V. Iakovenko, ${ }^{41}$ P. Ilten, ${ }^{12}$ J. Imong, ${ }^{43}$ R. Jacobsson, ${ }^{35}$ A. Jaeger, ${ }^{11}$ E. Jans,${ }^{38}$ F. Jansen, ${ }^{38}$ P. Jaton, ${ }^{36}$ F. Jing, ${ }^{3}$ M. John,${ }^{52}$ D. Johnson,${ }^{52}$ C. R. Jones,${ }^{44}$ B. Jost,${ }^{35}$ M. Kaballo, ${ }^{9}$ S. Kandybei, ${ }^{40}$ M. Karacson, ${ }^{35}$ T. M. Karbach, ${ }^{35}$ I. R. Kenyon, ${ }^{42}$ U. Kerzel, ${ }^{35}$ T. Ketel, ${ }^{39}$ A. Keune,${ }^{36}$ B. Khanji, ${ }^{20}$ O. Kochebina, ${ }^{7}$ I. Komarov, ${ }^{36,29}$ R. F. Koopman, ${ }^{39}$ P. Koppenburg, ${ }^{38}$ M. Korolev, ${ }^{29}$ A. Kozlinskiy,${ }^{38}$ L. Kravchuk, ${ }^{30}$

K. Kreplin, ${ }^{11}$ M. Kreps, ${ }^{45}$ G. Krocker, ${ }^{11}$ P. Krokovny, ${ }^{31}$ F. Kruse, ${ }^{9}$ M. Kucharczyk, ${ }^{20,23, \mathrm{e}}$ V. Kudryavtsev, ${ }^{31}$ T. Kvaratskheliya ${ }^{28,35}$ V. N. La Thi,${ }^{36}$ D. Lacarrere, ${ }^{35}$ G. Lafferty, ${ }^{51}$ A. Lai,${ }^{15}$ D. Lambert, ${ }^{47}$ R. W. Lambert,${ }^{39}$ E. Lanciotti, ${ }^{35}$ G. Lanfranchi, ${ }^{18,35}$ C. Langenbruch, ${ }^{35}$ T. Latham, ${ }^{45}$ C. Lazzeroni, ${ }^{42}$ R. Le Gac, ${ }^{6}$ J. van Leerdam, ${ }^{38}$ 
J.-P. Lees, ${ }^{4}$ R. Lefèvre, ${ }^{5}$ A. Leflat, ${ }^{29,35}$ J. Lefrançois, ${ }^{7}$ O. Leroy,${ }^{6}$ Y. Li,${ }^{3}$ L. Li Gioi, ${ }^{5}$ M. Liles,${ }^{49}$ R. Lindner, ${ }^{35}$ C. Linn, ${ }^{11}$ B. Liu, ${ }^{3}$ G. Liu, ${ }^{35}$ J. von Loeben, ${ }^{20}$ J. H. Lopes, ${ }^{2}$ E. Lopez Asamar, ${ }^{33}$ N. Lopez-March,${ }^{36}$ H. Lu, ${ }^{3}$ J. Luisier, ${ }^{36}$ H. Luo, ${ }^{47}$ A. Mac Raighne, ${ }^{48}$ F. Machefert, ${ }^{7}$ I. V. Machikhiliyan, ${ }^{4,28}$ F. Maciuc, ${ }^{26}$ O. Maev, ${ }^{27,35}$ S. Malde ${ }^{52}$ G. Manca, ${ }^{15, j}$ G. Mancinelli, ${ }^{6}$ N. Mangiafave, ${ }^{44}$ U. Marconi, ${ }^{14}$ R. Märki, ${ }^{36}$ J. Marks, ${ }^{11}$ G. Martellotti, ${ }^{22}$ A. Martens, ${ }^{8}$ L. Martin, ${ }^{52}$ A. Martín Sánchez,${ }^{7}$ M. Martinelli, ${ }^{38}$ D. Martinez Santos, ${ }^{39}$ D. Martins Tostes, ${ }^{2}$ A. Massafferri, ${ }^{1}$ R. Matev,${ }^{35}$ Z. Mathe,${ }^{35}$ C. Matteuzzi ${ }^{20}$ M. Matveev, ${ }^{27}$ E. Maurice, ${ }^{6}$ A. Mazurov, ${ }^{16,30,35, i}$ J. McCarthy, ${ }^{42}$ R. McNulty, ${ }^{12}$ B. Meadows,${ }^{57,52}$ F. Meier, ${ }^{9}$ M. Meissner, ${ }^{11}$ M. Merk, ${ }^{38}$ D. A. Milanes, ${ }^{13}$ M.-N. Minard, ${ }^{4}$ J. Molina Rodriguez, ${ }^{54}$ S. Monteil, ${ }^{5}$ D. Moran,${ }^{51}$ P. Morawski, ${ }^{23}$ R. Mountain, ${ }^{53}$ I. Mous, ${ }^{38}$ F. Muheim, ${ }^{47}$ K. Müller, ${ }^{37}$ R. Muresan, ${ }^{26}$ B. Muryn, ${ }^{24}$ B. Muster, ${ }^{36}$ P. Naik, ${ }^{43}$ T. Nakada, ${ }^{36}$ R. Nandakumar, ${ }^{46}$ I. Nasteva, ${ }^{1}$ M. Needham, ${ }^{47}$ N. Neufeld, ${ }^{35}$ A. D. Nguyen, ${ }^{36}$ T. D. Nguyen,${ }^{36}$ C. Nguyen-Mau, ${ }^{36, k}$ M. Nicol, ${ }^{7}$ V. Niess, ${ }^{5}$ R. Niet, ${ }^{9}$ N. Nikitin, ${ }^{29}$ T. Nikodem, ${ }^{11}$ S. Nisar ${ }^{56}$ A. Nomerotski, ${ }^{52}$ A. Novoselov, ${ }^{32}$

A. Oblakowska-Mucha, ${ }^{24}$ V. Obraztsov, ${ }^{32}$ S. Oggero, ${ }^{38}$ S. Ogilvy, ${ }^{48}$ O. Okhrimenko, ${ }^{41}$ R. Oldeman, ${ }^{15,35, j}$ M. Orlandea ${ }^{26}$ J. M. Otalora Goicochea, ${ }^{2}$ P. Owen,${ }^{50}$ B. K. Pal,${ }^{53}$ A. Palano, ${ }^{13,1}$ M. Palutan, ${ }^{18}$ J. Panman, ${ }^{35}$ A. Papanestis, ${ }^{46}$ M. Pappagallo, ${ }^{48}$ C. Parkes,${ }^{51}$ C. J. Parkinson, ${ }^{50}$ G. Passaleva,,${ }^{17}$ G. D. Patel,${ }^{49}$ M. Patel,${ }^{50}$ G. N. Patrick, ${ }^{46}$ C. Patrignani, ${ }^{19, h}$ C. Pavel-Nicorescu, ${ }^{26}$ A. Pazos Alvarez,${ }^{34}$ A. Pellegrino, ${ }^{38}$ G. Penso, ${ }^{22, m}$ M. Pepe Altarelli, ${ }^{35}$ S. Perazzini, ${ }^{14, f}$ D. L. Perego,${ }^{20, \mathrm{e}}$ E. Perez Trigo, ${ }^{34}$ A. Pérez-Calero Yzquierdo, ${ }^{33}$ P. Perret, ${ }^{5}$ M. Perrin-Terrin, ${ }^{6}$ G. Pessina, ${ }^{20}$ K. Petridis, ${ }^{50}$ A. Petrolini, ${ }^{19, h}$ A. Phan, ${ }^{53}$ E. Picatoste Olloqui, ${ }^{33}$ B. Pietrzyk ${ }^{4}$ T. Pilař, ${ }^{45}$ D. Pinci, ${ }^{22}$ S. Playfer, ${ }^{47}$ M. Plo Casasus, ${ }^{34}$ F. Polci, ${ }^{8}$ G. Polok, ${ }^{23}$ A. Poluektov, ${ }^{45,31}$ E. Polycarpo, ${ }^{2}$ D. Popov, ${ }^{10}$ B. Popovici ${ }^{26}$ C. Potterat,${ }^{33}$ A. Powell,${ }^{52}$ J. Prisciandaro, ${ }^{36}$ V. Pugatch,${ }^{41}$ A. Puig Navarro, ${ }^{36}$ W. Qian, ${ }^{4}$ J. H. Rademacker, ${ }^{43}$ B. Rakotomiaramanana, ${ }^{36}$ M. S. Rangel, ${ }^{2}$ I. Raniuk, ${ }^{40}$ N. Rauschmayr, ${ }^{35}$ G. Raven, ${ }^{39}$

S. Redford, ${ }^{52}$ M. M. Reid, ${ }^{45}$ A. C. dos Reis, ${ }^{1}$ S. Ricciardi, ${ }^{46}$ A. Richards,${ }^{50}$ K. Rinnert, ${ }^{49}$ V. Rives Molina, ${ }^{33}$ D. A. Roa Romero, ${ }^{5}$ P. Robbe, ${ }^{7}$ E. Rodrigues,${ }^{51}$ P. Rodriguez Perez,${ }^{34}$ G. J. Rogers, ${ }^{44}$ S. Roiser, ${ }^{35}$ V. Romanovsky, ${ }^{32}$ A. Romero Vidal, ${ }^{34}$ J. Rouvinet, ${ }^{36}$ T. Ruf,${ }^{35}$ H. Ruiz, ${ }^{33}$ G. Sabatino, ${ }^{22, g}$ J. J. Saborido Silva,${ }^{34}$ N. Sagidova,${ }^{27}$ P. Sail, ${ }^{48}$ B. Saitta, ${ }^{15, j}$ C. Salzmann, ${ }^{37}$ B. Sanmartin Sedes,${ }^{34}$ M. Sannino, ${ }^{19, h}$ R. Santacesaria,${ }^{22}$ C. Santamarina Rios, ${ }^{34}$ E. Santovetti, ${ }^{21, \mathrm{~g}}$ M. Sapunov, ${ }^{6}$ A. Sarti, ${ }^{18, \mathrm{~m}}$ C. Satriano, ${ }^{22, \mathrm{c}}$ A. Satta, ${ }^{21}$ M. Savrie, ${ }^{16, i}$ D. Savrina, ${ }^{28,29}$ P. Schaack, ${ }^{50}$ M. Schiller, ${ }^{39}$ H. Schindler, ${ }^{35}$ S. Schleich, ${ }^{9}$ M. Schlupp,,${ }^{9}$ M. Schmelling, ${ }^{10}$ B. Schmidt,${ }^{35}$ O. Schneider, ${ }^{36}$ A. Schopper, ${ }^{35}$ M.-H. Schune, ${ }^{7}$ R. Schwemmer, ${ }^{35}$ B. Sciascia, ${ }^{18}$ A. Sciubba, ${ }^{18, m}$ M. Seco, ${ }^{34}$ A. Semennikov, ${ }^{28}$ K. Senderowska, ${ }^{24}$ I. Sepp,${ }^{50}$ N. Serra, ${ }^{37}$ J. Serrano, ${ }^{6}$ P. Seyfert, ${ }^{11}$ M. Shapkin, ${ }^{32}$ I. Shapoval,${ }^{40,35}$ P. Shatalov, ${ }^{28}$ Y. Shcheglov, ${ }^{27}$ T. Shears, ${ }^{49,35}$ L. Shekhtman, ${ }^{31}$ O. Shevchenko, ${ }^{40}$ V. Shevchenko, ${ }^{28}$ A. Shires,${ }^{50}$ R. Silva Coutinho, ${ }^{45}$ T. Skwarnicki, ${ }^{53}$ N. A. Smith, ${ }^{49}$ E. Smith,${ }^{52,46}$ M. Smith, ${ }^{51}$ K. Sobczak ${ }^{5}$ M. D. Sokoloff, ${ }^{57}$ F. J. P. Soler ${ }^{48}$

F. Soomro, ${ }^{18,35}$ D. Souza ${ }^{43}$ B. Souza De Paula, ${ }^{2}$ B. Spaan, ${ }^{9}$ A. Sparkes,${ }^{47}$ P. Spradlin,${ }^{48}$ F. Stagni, ${ }^{35}$ S. Stahl, ${ }^{11}$ O. Steinkamp, ${ }^{37}$ S. Stoica, ${ }^{26}$ S. Stone,${ }^{53}$ B. Storaci,${ }^{37}$ M. Straticiuc, ${ }^{26}$ U. Straumann, ${ }^{37}$ V. K. Subbiah, ${ }^{35}$ S. Swientek,${ }^{9}$ V. Syropoulos, ${ }^{39}$ M. Szczekowski, ${ }^{25}$ P. Szczypka, ${ }^{36,35}$ T. Szumlak, ${ }^{24}$ S. T' Jampens, ${ }^{4}$ M. Teklishyn, ${ }^{7}$ E. Teodorescu, ${ }^{26}$

F. Teubert,${ }^{35}$ C. Thomas, ${ }^{52}$ E. Thomas,${ }^{35}$ J. van Tilburg, ${ }^{11}$ V. Tisserand,${ }^{4}$ M. Tobin,${ }^{37}$ S. Tolk, ${ }^{39}$ D. Tonelli, ${ }^{35}$ S. Topp-Joergensen,${ }^{52} \mathrm{~N}$. Torr, ${ }^{52}$ E. Tournefier, ${ }^{4,50} \mathrm{~S}$. Tourneur, ${ }^{36} \mathrm{M}$. T. Tran, ${ }^{36} \mathrm{M}$. Tresch, ${ }^{37}$ A. Tsaregorodtsev, ${ }^{6}$ P. Tsopelas, ${ }^{38}$ N. Tuning, ${ }^{38}$ M. Ubeda Garcia, ${ }^{35}$ A. Ukleja, ${ }^{25}$ D. Urner,${ }^{51}$ U. Uwer, ${ }^{11}$ V. Vagnoni, ${ }^{14}$ G. Valenti, ${ }^{14}$

R. Vazquez Gomez,${ }^{33}$ P. Vazquez Regueiro, ${ }^{34}$ S. Vecchi, ${ }^{16}$ J. J. Velthuis, ${ }^{43}$ M. Veltri, ${ }^{17, n}$ G. Veneziano, ${ }^{36}$ M. Vesterinen ${ }^{35}$ B. Viaud, ${ }^{7}$ D. Vieira, ${ }^{2}$ X. Vilasis-Cardona, ${ }^{33, a}$ A. Vollhardt,${ }^{37}$ D. Volyanskyy, ${ }^{10}$ D. Voong, ${ }^{43}$

A. Vorobyev, ${ }^{27}$ V. Vorobyev, ${ }^{31}$ C. Voß,${ }^{55}$ H. Voss,${ }^{10}$ R. Waldi, ${ }^{55}$ R. Wallace,,${ }^{12}$ S. Wandernoth, ${ }^{11}$ J. Wang,${ }^{53}$ D. R. Ward ${ }^{44}$ N. K. Watson, ${ }^{42}$ A. D. Webber, ${ }^{51}$ D. Websdale, ${ }^{50}$ M. Whitehead, ${ }^{45}$ J. Wicht, ${ }^{35}$ J. Wiechczynski, ${ }^{23}$ D. Wiedner ${ }^{11}$ L. Wiggers,${ }^{38}$ G. Wilkinson,${ }^{52}$ M. P. Williams, ${ }^{45,46}$ M. Williams,${ }^{50, o}$ F. F. Wilson, ${ }^{46}$ J. Wishahi, ${ }^{9}$ M. Witek ${ }^{23}$ W. Witzeling, ${ }^{35}$ S. A. Wotton ${ }^{44}$ S. Wright,${ }^{44}$ S. Wu, ${ }^{3}$ K. Wyllie, ${ }^{35}$ Y. Xie, ${ }^{47,35}$ F. Xing, ${ }^{52}$ Z. Xing, ${ }^{53}$ Z. Yang, ${ }^{3}$ R. Young, ${ }^{47}$ X. Yuan, ${ }^{3}$ O. Yushchenko, ${ }^{32}$ M. Zangoli, ${ }^{14}$ M. Zavertyaev, ${ }^{10,58}$ F. Zhang, ${ }^{3}$ L. Zhang, ${ }^{53}$ W. C. Zhang, ${ }^{12}$ Y. Zhang, ${ }^{3}$ A. Zhelezov, ${ }^{11}$ A. Zhokhov, ${ }^{28}$ L. Zhong, ${ }^{3}$ and A. Zvyagin ${ }^{35}$

(LHCb Collaboration)

\author{
${ }^{1}$ Centro Brasileiro de Pesquisas Físicas (CBPF), Rio de Janeiro, Brazil \\ ${ }^{2}$ Universidade Federal do Rio de Janeiro (UFRJ), Rio de Janeiro, Brazil \\ ${ }^{3}$ Center for High Energy Physics, Tsinghua University, Beijing, China \\ ${ }^{4} L A P P$, Université de Savoie, CNRS/IN2P3, Annecy-Le-Vieux, France \\ ${ }^{5}$ Clermont Université, Université Blaise Pascal, CNRS/IN2P3, LPC, Clermont-Ferrand, France
}


${ }^{6}$ CPPM, Aix-Marseille Université, CNRS/IN2P3, Marseille, France

${ }^{7}$ LAL, Université Paris-Sud, CNRS/IN2P3, Orsay, France

${ }^{8}$ LPNHE, Université Pierre et Marie Curie, Université Paris Diderot, CNRS/IN2P3, Paris, France

${ }^{9}$ Fakultät Physik, Technische Universität Dortmund, Dortmund, Germany

${ }^{10}$ Max-Planck-Institut für Kernphysik (MPIK), Heidelberg, Germany

${ }^{11}$ Physikalisches Institut, Ruprecht-Karls-Universität Heidelberg, Heidelberg, Germany

${ }^{12}$ School of Physics, University College Dublin, Dublin, Ireland

${ }^{13}$ Sezione INFN di Bari, Bari, Italy

${ }^{14}$ Sezione INFN di Bologna, Bologna, Italy

${ }^{15}$ Sezione INFN di Cagliari, Cagliari, Italy

${ }^{16}$ Sezione INFN di Ferrara, Ferrara, Italy

${ }^{17}$ Sezione INFN di Firenze, Firenze, Italy

${ }^{18}$ Laboratori Nazionali dell'INFN di Frascati, Frascati, Italy

${ }^{19}$ Sezione INFN di Genova, Genova, Italy

${ }^{20}$ Sezione INFN di Milano Bicocca, Milano, Italy

${ }^{21}$ Sezione INFN di Roma Tor Vergata, Roma, Italy

${ }^{22}$ Sezione INFN di Roma La Sapienza, Roma, Italy

${ }^{23}$ Henryk Niewodniczanski Institute of Nuclear Physics Polish Academy of Sciences, Kraków, Poland

${ }^{24}$ AGH University of Science and Technology, Kraków, Poland

${ }^{25}$ National Center for Nuclear Research (NCBJ), Warsaw, Poland

${ }^{26}$ Horia Hulubei National Institute of Physics and Nuclear Engineering, Bucharest-Magurele, Romania

${ }^{27}$ Petersburg Nuclear Physics Institute (PNPI), Gatchina, Russia

${ }^{28}$ Institute of Theoretical and Experimental Physics (ITEP), Moscow, Russia

${ }^{29}$ Institute of Nuclear Physics, Moscow State University (SINP MSU), Moscow, Russia

${ }^{30}$ Institute for Nuclear Research of the Russian Academy of Sciences (INR RAN), Moscow, Russia

${ }^{31}$ Budker Institute of Nuclear Physics (SB RAS) and Novosibirsk State University, Novosibirsk, Russia

${ }^{32}$ Institute for High Energy Physics (IHEP), Protvino, Russia

${ }^{33}$ Universitat de Barcelona, Barcelona, Spain

${ }^{34}$ Universidad de Santiago de Compostela, Santiago de Compostela, Spain

${ }^{35}$ European Organization for Nuclear Research (CERN), Geneva, Switzerland

${ }^{36}$ Ecole Polytechnique Fédérale de Lausanne (EPFL), Lausanne, Switzerland

${ }^{37}$ Physik-Institut, Universität Zürich, Zürich, Switzerland

${ }^{38}$ Nikhef National Institute for Subatomic Physics, Amsterdam, The Netherlands

${ }^{39}$ Nikhef National Institute for Subatomic Physics and VU University Amsterdam, Amsterdam, The Netherlands

${ }^{40}$ NSC Kharkiv Institute of Physics and Technology (NSC KIPT), Kharkiv, Ukraine

${ }^{41}$ Institute for Nuclear Research of the National Academy of Sciences (KINR), Kyiv, Ukraine

${ }^{42}$ University of Birmingham, Birmingham, United Kingdom

${ }^{43}$ H. H. Wills Physics Laboratory, University of Bristol, Bristol, United Kingdom

${ }^{44}$ Cavendish Laboratory, University of Cambridge, Cambridge, United Kingdom

${ }^{45}$ Department of Physics, University of Warwick, Coventry, United Kingdom

${ }^{46}$ STFC Rutherford Appleton Laboratory, Didcot, United Kingdom

${ }^{47}$ School of Physics and Astronomy, University of Edinburgh, Edinburgh, United Kingdom

${ }^{48}$ School of Physics and Astronomy, University of Glasgow, Glasgow, United Kingdom

${ }^{49}$ Oliver Lodge Laboratory, University of Liverpool, Liverpool, United Kingdom

${ }^{50}$ Imperial College London, London, United Kingdom

${ }^{51}$ School of Physics and Astronomy, University of Manchester, Manchester, United Kingdom

${ }^{52}$ Department of Physics, University of Oxford, Oxford, United Kingdom

${ }^{53}$ Syracuse University, Syracuse, NY, USA

${ }^{54}$ Pontifícia Universidade Católica do Rio de Janeiro (PUC-Rio), Rio de Janeiro,

Brazil (associated to Universidade Federal do Rio de Janeiro (UFRJ), Rio de Janeiro, Brazil)

${ }^{55}$ Institut für Physik, Universität Rostock, Rostock, Germany (associated to Physikalisches Institut,

Ruprecht-Karls-Universität Heidelberg, Heidelberg, Germany)

${ }^{56}$ Institute of Information Technology, COMSATS, Lahore, Pakistan (associated to Syracuse University, Syracuse, NY, USA)

${ }^{57}$ University of Cincinnati, Cincinnati, OH, United States (associated to Syracuse University, Syracuse, NY, USA)

${ }^{58}$ P. N. Lebedev Physical Institute, Russian Academy of Science (LPI RAS), Moscow, Russia

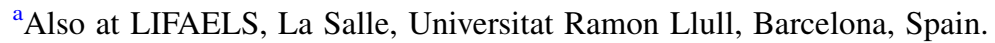

${ }^{\mathrm{b}}$ Also at Università di Firenze, Firenze, Italy.

${ }^{\mathrm{c}}$ Also at Università della Basilicata, Potenza, Italy.

${ }^{\mathrm{d} A l s o}$ at Università di Modena e Reggio Emilia, Modena, Italy. 
eAlso at Università di Milano Bicocca, Milano, Italy.

${ }^{\mathrm{f}}$ Also at Università di Bologna, Bologna, Italy.

${ }^{\mathrm{g}}$ Also at Università di Roma Tor Vergata, Roma, Italy.

${ }^{\mathrm{h}}$ Also at Università di Genova, Genova, Italy.

${ }^{\mathrm{i}}$ Also at Università di Ferrara, Ferrara, Italy.

${ }^{j}$ Also at Università di Cagliari, Cagliari, Italy.

${ }^{\mathrm{k}}$ Also at Hanoi University of Science, Hanoi, Viet Nam.

${ }^{1}$ Also at Università di Bari, Bari, Italy.

${ }^{\mathrm{m}}$ Also at Università di Roma La Sapienza, Roma, Italy.

${ }^{\mathrm{n}}$ Also at Università di Urbino, Urbino, Italy.

${ }^{\circ}$ Also at Massachusetts Institute of Technology, Cambridge, MA, USA. 